\title{
Shells of dust around AGB stars: Effects on the integrated spectrum of Single Stellar Populations
}

\begin{abstract}
L. Piovan, R. Tantalo, and C. Chiosi
Department of Astronomy, University of Padova, Vicolo dell'Osservatorio 2, 35122 Padova, Italy

e-mail: tantalo@pd.astro.it; chiosi@pd.astro.it

Received 20 January 2003 / Accepted 18 June 2003

Abstract. In this paper we present models for Single Stellar Populations (SSPs) of intermediate and old ages where dust enshrouded Asymptotic Giant Branch (AGB) stars are introduced. As long known AGB stars are surrounded by dust-rich shells of matter caused by their own stellar wind, which absorb the radiation coming from the central object and re-emit it in the far infrared (IR). To this aim, particular care is devoted to follow the evolution of the AGB stars throughout the quiet and thermally pulsing regimes, to evaluate the effect of self contamination in the outermost layers by the third dredge-up mechanism, to follow the transition from oxygen-rich to carbon-rich objects (as appropriate to their initial mass and chemical composition), and finally to estimate the efficiency of mass-loss by stellar winds, all aspects that concur to the formation and properties of the dusty shells around. In addition to this, accurate physical models of the dusty shells are presented in which the re-processing of radiation from the central stars is calculated by solving the radiative transfer equations in presence of dust particles of different chemical composition. The resulting spectral energy distribution (SED) is examined to show how important features, like the $10 \mu \mathrm{m} \mathrm{Si}-\mathrm{O}$ stretching mode feature and the $11 \mu \mathrm{m} \mathrm{SiC}$ feature, evolve with time. The SEDs are then convolved with the IRAS filters to obtain the flux in various pass-bands, i.e. 12, 25 and $60 \mu \mathrm{m}$, for individual AGB stars of different mass, chemical composition, and age. The comparison is made by means of SSPs along which AGB stars of the same age but different initial masses are located. This allows us to explore the whole range of masses and ages spanned by AGB stars. The theoretical results are compared to the observational data for selected groups of stars. The same is made for the $J, H, K, L$ pass-bands of the Johnson system. Finally, from the integrated SEDs of the SSPs, we derive the integrated Johnson $J, H, K, L$ magnitudes and colors to be compared to infrared data for star clusters of the Magellanic Clouds. In general good agreement with the data is possible if the effects of the circumstellar shells of dust are taken into account.
\end{abstract}

Key words. stars: AGB and post AGB - stars: mass-loss - stars: circumstellar matter - infrared: stars

\section{Introduction}

The first all-sky survey at far-IR wavelengths carried out in 1983 by the Infrared Astronomical Satellite (IRAS) has opened a new era of modern infrared astronomy. Thousands of galaxies were detected to emit most of their light in the IR. After IRAS, a long series of observations were started to explore the IR Universe. In the COBE all-sky maps a bright isotropic background (CIRB) was discovered in the far-IR/sub$\mathrm{mm}$ spectral regions, whose origin is nowadays attributed to the integrated emission by dust in primeval galaxies absorbing and scattering the stellar light and returning it at long wavelengths (Puget et al. 1996). For the first time, the Infrared Space Observatory (ISO) surveyed and detected distant galaxies in the mid- and far-IR, allowing us to know in detail their emission in those spectral regions (Elbaz et al. 1999).

It became soon clear that precious information on the star formation history (SFH) of galaxies and the Universe

Send offprint requests to: L. Piovan,

e-mail: piovan@pd.astro.it as a whole is hidden in the UV-optical and IR ranges of the spectral energy distribution, thus spurring cross-correlated studies of the two regions. Many wide-field and all-sky surveys are currently running or have been just completed, e.g. the Galaxy Evolution Explorer [GALEX] (Martin et al. 1997) and the Sloan Digital Sky Survey [SDSS] (York et al. 2000) in the UV-optical range; the Two Micron All-Sky Survey [2MASS] (Skrutskie et al. 1997) and the Deep NearInfrared Survey of the Southern Sky [DENIS] (Epchtein et al. 1997) in the near infrared. Combined with other astronomical databases, they provide a huge amount of UV-optical and near IR photometric data for millions of galaxies. In particular, the observational information in the infrared will further grow with the new surveys SIRTF (Space Infrared Telescope Facility, see http://sirtf.caltech.edu/SSC/), UKIDSS (the successor of 2MASS, see http://www.ukidss.org/) and with the advent of the Next Generation Space Telescope (NGST/JWST).

This wealth of data must be accompanied by a continuous upgrade of the basic theoretical tools to fully exploit any 
information on the physical properties of the observed objects. In particular, to understand the role played by dust as strong IR emitter is mandatory. The key instruments for photometric studies are the SSPs, the building blocks of the assemblies of stars of different complexity going from star clusters to galaxies, and the fundamentals of population synthesis techniques. An SSP is defined as a coeval, chemically homogeneous assembly of stars all contributing to build up the integrated SED in a way proportional to the duration of their evolutionary phase, luminosity and relative number (the luminosity function), in other words according to the precepts of Fuel Consumption Theorem (Tinsley 1980; Renzini \& Buzzoni 1983).

Let us shortly summarize here the various steps by which the theoretical SED of an SSP is derived, (a) given an age, the corresponding isochrone in the HRD is divided in elemental intervals small enough to assure that the luminosity, gravity, and $T_{\text {eff }}$ in them are nearly constant (the isochrone is approximated to series of virtual stars, for each of which we know the spectrum); (b) in each elemental interval the star mass spans a suitable range $\Delta M$ fixed by the evolutionary rate, therefore the number of stars per elemental interval is proportional to the integral of the initial mass function (IMF) over the range $\Delta M$ (the differential luminosity function); (c) finally, the contribution to the flux at each wavelength of the spectrum by each elemental interval is weighed on the number of stars in it and their luminosity. To conclude, basic ingredients of the SSP SEDs are the isochrones and their path in HRD (in turn functions of the initial chemical composition), the IMF, and a library of stellar spectra for different values of $T_{\text {eff }}$, gravity, and chemical composition.

With a few exceptions to be mentioned later, the light emitted by SSPs is modeled neglecting the presence of dust around their stars (e.g. Bertelli et al. 1994; Tantalo 1998; Girardi et al. 2002). However, there are at least two situations in which dust is present: the very initial stages when stars are born embedded in molecular clouds, and the late stages of AGB stars when dusty envelopes are formed. In this study we will concentrate on the AGB stars leaving to a forthcoming paper the case of young stars.

The dust shells surrounding AGB stars are the result of mass-loss by stellar winds and the complex structure and evolution of these stars during the thermally pulsing AGB phase (TP-AGB). They trap the radiation coming from the central AGB star and re-emit it in the far IR (see Habing 1996, for a classical review of this topic). The contribution to the IR radiation by the dusty shells of AGB stars is particularly relevant because AGB stars are luminous objects able to significantly affect the integrated SED of star clusters and galaxies.

A great deal of studies have been devoted to model the circumstellar shells around AGB stars and to understand in detail important properties of the shell structure, such as the composition of the dust grains, the dust-to-gas ratio, the expansion velocity of the matter, and the mass-loss rates (cf. Habing 1996). However, only an handful of studies have tried to include the effect of the dust shells surrounding AGB stars on the integrated SEDs of SSPs: the pioneer paper by Bressan et al. (1998) and the recent series of three articles by Mouhcine \& Lançon (2002); Lançon \& Mouhcine (2002) and Mouhcine (2002).
There are several causes hampering detailed studies of the subject:

(i) first of all, theoretical spectra of oxygen-rich (O-stars) and carbon-rich (C-stars) AGB stars, surrounded by shells of matter and even of simple AGB stars are not available. Only empirical spectra are to our disposal. This is an obvious difficulty for any fully theoretical analysis of the problem. The spectra of AGB stars of any type are particularly difficult to obtain because of the many parameters entering the problem, i.e. the spectrum of the inner stellar source, the optical depth (key parameter of the radiative transfer problem to be solved to follow the propagation of radiation throughout the dusty shell), and the properties of dust grains in the shell. These latter depend on the optical depth in such a way that we have different compositions for different optical depths and of course passing from O-stars to C-stars.

(ii) Second, for long time SSPs have been developed to interpret observational data in the optical range of the spectrum. Only recently the wealthy of data in the IR have spurred several groups to develop suitable SSPs extending to the far infrared. The study of Bressan et al. (1998), despite severe limitations such as the use of giant spectra to model the radiative transport in O-stars and C-stars, the lack of the transition between $\mathrm{O}$-stars and C-stars, and the crude treatment of the dust composition, has opened the way to a new generation of SSPs in the far IR. Mouhcine \& Lançon (2002) and Mouhcine (2002) have improved the situation including appropriate models for the AGB phase, the transition between O-stars and C-stars, and an empirical library of spectra of long period variables (LPV) as input for the circumstellar shells. It is important to remind here that while Bressan et al. (1998) made use of purely theoretical spectra, Mouhcine \& Lançon (2002) and Mouhcine (2002) have adopted an empirical library of spectra of AGB stars, so that part of their results is implicit in this initial assumption.

Aim of this study is to go further along the theoretical line of work and to generate modern integrated SEDs of SSPs that: (i) extend to the far IR; (ii) include more updated models of AGB stars; (iii) allow for the metallicity dependence and finally (iv) include an accurate modelling of the shells of dust surrounding these stars, thus improving upon the emission in the IR spectral region. We will follow a purely theoretical approach as in Bressan et al. (1998) and will try to include a detailed treatment of the circumstellar dusty shells and of the transition from $\mathrm{O}$ - to $\mathrm{C}$-stars. It is clear that the theoretical spectra of $\mathrm{M}$ giant stars are not as good as the empirical ones (they can indeed be applied only to the case of unobscured $\mathrm{O}$ - and C-stars). However, we will insist on the purely theoretical approach in order to establish how far current theory can go in interpreting and reproducing the observational data.

This paper is organized in the following way. In Sect. 2 we present the model for the envelope of AGB stars, describe the assumptions for the radiative transfer problem, and calculate an expression for the optical depth, the key parameter of the radiative transfer equations, and its dependence on basic stellar parameters such as the star mass $M$, radius $R$, effective temperature $T_{\text {eff }}$, luminosity $L$, pulsation period $P$, and metal content $Z$. In Sect. 3 we briefly summarize the prescription we have adopted for the mass-loss rates of AGB stars. In Sect. 4, 
we discuss in detail the optical properties of dusty AGB shells. As a matter of fact, the dust absorption coefficients are different for O-stars and C-stars, and even for the same type of stars, different optical depths may imply different properties. In Sect. 5 we present our choice for the parameters governing the dusty shells of AGB stars, i.e, the temperature on the inner boundary, the density profile across the shells, and the type and mixture of grains. In Sect. 6 we try to discuss and evaluate the uncertainty affecting the models of the dust shells and how this would reflect onto the SED of the SSPs with particular attention to the IR range. In Sect. 7 we define the monochromatic flux emitted by a SSP, summarize the assumption for the initial mass function and the rates of mass-loss from stars that we have adopted, report on the libraries of stellar models, isochrones and stellar spectra with aid of which we have calculated our SSPs, and finally discuss in detail the problem of the transition between O-stars and C-stars AGB stars and how this is incorporated in our SSPs. In particular we describe how we have included the recent models by Marigo et al. (1999) into the SSP models by Tantalo (1998). In Sect. 8 we present the SEDs of our new SSPs that take the effect of dusty AGB shells into account and compare our results with those for the standard SSPs (Tantalo 1998). In Sect. 9 we describe the integrated IRAS far IR colors predicted by our models and compare them with the observational data for a sample of AGB stars (Mira, Semi-Regular Variables, Long Period Variables, OH/IR stars and C-stars). We also derive the integrated near IR colors of the SSPs and compare them with the data for a selected sample of young star clusters of the Magellanic Clouds. In addition to this, we examine the age dependence of the integrated near IR colors of SSPs from different sources, i.e. Bertelli et al. (1994); Tantalo (1998); Girardi et al. (2002); Mouhcine \& Lançon (2002); Lançon \& Mouhcine (2002) and this study. Finally, some concluding remarks are drawn in Sect. 10.

\section{Modelling a dusty envelope}

The problem of radiative transfer in the dusty shells surrounding the mass losing stars (AGB stars in particular) has been addressed by many authors (see Habing 1996, and references therein). The most complete formulation of the problem couples the radiative transfer and hydro-dynamical equations for the motion of the two interacting fluids, gas and dust, and takes the interdependence between gas, dust and radiation pressure into account.

Since our goal is to simply include the effects of the AGB star dusty shells on the spectra of SSPs, we will limit ourselves to consider the problem of radiative transfer in the shell and will leave hydrodynamics aside. To this aim, we need to link the fundamental parameters of a star, i.e. mass $M$, radius $R$, luminosity $L$, pulsational period $P$, and metal abundance $Z$ to the parameters that characterize the shell of matter and that are relevant to the solution of the radiative transfer across it.

The radiative transfer equation is

$\frac{\mathrm{d} I_{\lambda}(l)}{\mathrm{d} l}=k_{\lambda}(l)\left[S_{\lambda}(l)-I_{\lambda}(l)\right]$ where

- $k_{\lambda}(l)$ is the overall extinction coefficient at the wavelength $\lambda$ given by the sum of the absorption and scattering coefficients, $k_{\lambda}(l)=k_{\mathrm{a} \lambda}(l)+k_{\mathrm{s} \lambda}(l)$;

- $I_{\lambda}(l)$ is the intensity of the radiation field;

- $S_{\lambda}(l)$ is the "source function", given by the ratio $\varepsilon_{\lambda}(l) / k_{\lambda}(l)$, with $\varepsilon_{\lambda}(l)$ the emission coefficient.

As demonstrated by Rowan-Robinson (1980) and Ivezic \& Elitzur $(1995,1997)$, only two dimensional scales are involved in the radiative transfer equation because $I_{\lambda}$ and $S_{\lambda}$ have the dimension of an intensity, whereas $k_{\lambda}$ and $l$ have the dimension of a length ( $k_{\lambda}$ is the inverse of a length). Therefore, any physical quantity related to the radiative transfer problem can be expressed as functions of these two scales.

Defining the dimensionless element of optical depth along the ray path $\mathrm{d} l$ as $\mathrm{d} \tau_{\lambda}=k_{\lambda}(l) \mathrm{d} l$, we can write Eq. (1) as

$\frac{\mathrm{d} I_{\lambda}(l)}{\mathrm{d} \tau_{\lambda}}=S_{\lambda}(l)-I_{\lambda}(l)$.

Starting from these simple considerations, Ivezic \& Elitzur (1997) have proved that the radiative transfer equation satisfies the property of scale invariance: the physical dimension of any system can be increased and decreased in an arbitrary way without affecting the radiative properties, as long as optical depths and spatial variation of the opacity remain the same. Two systems with different dimensions and absorption coefficients, but with the same total optical depths and auto-similar distributions of opacities and "source functions" will produce the same intensity of the radiation field.

Rowan-Robinson (1980) first applied the radiative transfer scale invariance to the IR emission of a central source surrounded by a dust shell. Subsequently, Ivezic \& Elitzur (1997) presented a general formulation of the problem in arbitrary geometry and distribution of dust, and studied in detail the case of a spherical shell of dust heated up by a central source. They also pointed out that the concept of scale invariance is particularly useful when the absorption coefficient does not depend on the radiation intensity. Unfortunately, as a consequence of this, the analysis by Ivezic \& Elitzur (1997) cannot be applied to emission or photoionization lines (where the absorption coefficient can depend on the intensity of the radiation field via its effects on the level populations) but only to the continuum of the radiation coming from dust heated by a central source. Last point to note is that when the shell is optically thin over all wavelengths $\lambda$, the approximation $\tau_{\lambda} \sim 0$ may be used thus obtaining a quick analytical solution of the problem. In contrast, when the shell is optically thick the problem must be solved numerically.

In the following we apply the method, the results and the numerical code DUSTY by Ivezic \& Elitzur (1997) to study the dust shells surrounding AGB stars. The spherical symmetry approximation is adopted for the sake of simplicity. The key parameter we need to solve the radiative transfer problem and to calculate the emerging flux is the optical depth $\mathcal{T}_{\lambda}$ of the shell which is defined as follows

$\mathcal{T}_{\lambda}=\int_{S} \mathrm{~d} \tau_{\lambda}(r)=\int_{S} k_{\lambda}(r) \rho(r) \mathrm{d} r$ 
where $k_{\lambda}$ is the overall extinction coefficient per mass unit, $\rho$ is the matter density. Both depend on the radial distance $r$ from the central source. The integral is evaluated over the thickness $S$ of the shell. In the case of a dusty shell Eq. (3) becomes

$\mathcal{T}_{\lambda}=\int_{S} k_{\lambda}(r) \rho_{\mathrm{d}}(r) \mathrm{d} r$

where $\rho_{\mathrm{d}}(r)$ is the dust density.

The equation of mass conservation is given by

$\frac{\mathrm{d} M(r)}{\mathrm{d} t}=4 \pi r^{2} \rho(r) v(r)$.

The matter density $\rho(r)$ is linked to the dust density $\rho_{\mathrm{d}}(r)$ by the relation $\rho_{\mathrm{d}}(r)=\rho(r) \delta$ where $\delta$ is the dust-to-gas ratio. Substituting the matter density $\rho$ with the dust density $\rho_{\mathrm{d}}$ we obtain

$\frac{\mathrm{d} M(r)}{\mathrm{d} t}=\frac{4 \pi r^{2} \rho_{\mathrm{d}}(r) v(r)}{\delta}$

and then

$\rho_{\mathrm{d}}(r)=\frac{\dot{M}(r) \delta}{4 \pi r^{2} v(r)}$.

With aid of the above relation we recast the optical depth of Eq. (4) as

$\mathcal{T}_{\lambda}=\int_{S} k_{\lambda}(r) \frac{\dot{M}(r) \delta}{4 \pi r^{2} v(r)} \mathrm{d} r$.

In reality, the dusty shell will extend between an internal and external radius, $r_{\text {in }}$ and $r_{\text {out }}$ respectively. Assuming $\delta$ to be constant across the shell, we get

$\mathcal{T}_{\lambda}=\frac{\delta}{4 \pi} \int_{r_{\text {in }}}^{r_{\text {out }}} k_{\lambda}(r) \frac{\dot{M}(r)}{r^{2} v(r)} \mathrm{d} r$.

To proceed further, the mass-loss rate $\dot{M(r)}$, velocity $v(r)$, and extinction coefficient $k_{\lambda}(r)$ and their radial dependence must be specified. To a first approximation we assume that at any given time the rate of mass-loss and the velocity are constant with $r$, together with physical properties of the dust. Therefore the absorption coefficient for unit mass $k_{\lambda}$ does not depend on the radial coordinate $r$. With these simplifications we have

$\mathcal{T}_{\lambda}=\frac{\delta \dot{M} k_{\lambda}}{4 \pi v} \int_{r_{\text {in }}}^{r_{\text {out }}} \frac{1}{r^{2}} \mathrm{~d} r$

and upon integration

$\mathcal{T}_{\lambda}=\frac{\delta \dot{M} k_{\lambda}}{4 \pi v}\left(\frac{1}{r_{\text {in }}}-\frac{1}{r_{\text {out }}}\right)$.

Since $r_{\text {out }}$ is usually much larger than $r_{\text {in }}$, the relation above can be safely approximated to

$\mathcal{T}_{\lambda} \simeq \frac{\delta \dot{M} k_{\lambda}}{4 \pi v} \frac{1}{r_{\mathrm{in}}}$

Now we need to connect the physical quantities defining the optical depth of the dusty shell with the typical parameters of AGB stars, e.g. mass $M$, radius $R$, effective temperature $T_{\text {eff }}$, period $P$ of pulsation, luminosity $L$, and metal content $Z$.
Assuming the shell to be optically thick to IR radiation (a good approximation in case of high mass-loss rates), the inner radius of the shell can be derived from the equality

$L=4 \pi R_{*}^{2} \sigma T_{\mathrm{eff}}^{4}=4 \pi r_{\mathrm{in}}^{2} \sigma T_{\mathrm{d}}^{4}$

where $R_{*}$ is the stellar radius and $T_{\mathrm{d}}$ is the temperature of dust condensation at $r_{\text {in }}$. From this relation we get

$r_{\text {in }}=\left(\frac{L_{\odot}}{4 \pi \sigma T_{\mathrm{d}}^{4}}\right)^{\frac{1}{2}}\left(\frac{L}{L_{\odot}}\right)^{\frac{1}{2}}$.

The uncertainty arising from using the relation (13) can be transferred to $T_{\mathrm{d}}$ which is not firmly established. Literature values range from 800 to 1500 (Rowan-Robinson \& Harris 1982; David \& Papoular 1990; Suh 1999, 2000; Lorenz-Martins \& Pompeia 2000; Lorenz-Martins et al. 2001; Suh 2002). Finally, adopting $T_{\mathrm{d}}=1000 \mathrm{~K}$ (see Sect. 5 below) we obtain

$r_{\text {in }}=2.37 \times 10^{12}\left(\frac{L}{L_{\odot}}\right)^{\frac{1}{2}} \mathrm{~cm}$.

The extinction coefficient for unit mass $k_{\lambda}$ is in general given by

$k_{\lambda}=\frac{\sum_{i} n_{\mathrm{g}_{i}} \sigma_{\mathrm{g}_{i}}}{\rho_{\mathrm{d}}}$

where the summation is extended over all types of grain in the mixture, $\sigma_{\mathrm{g}_{i}}$ is the cross section for the $i$ th type of dust grain and $n_{\mathrm{g}_{i}}$ is the number of grains of $i$ th type for unit volume. Denoting with $m_{\mathrm{g}_{i}}$ the mass of the $i$ th type of dust grains, and introducing the mass abundance $\chi_{i}=n_{\mathrm{g}_{i}} m_{\mathrm{g}_{i}} / \rho_{\mathrm{d}}$ of $i$ th type of grain we finally obtain

$k_{\lambda}=\sum_{i} \chi_{i} \frac{\sigma_{\mathrm{g}_{i}}}{m_{\mathrm{g}_{i}}}$

The optical depth $\mathcal{T}_{\lambda}$ of the dust is a function of $k_{\lambda}$, and $k_{\lambda}$ in turn is a function of $\mathcal{T}_{\lambda}$ via the mass abundance $\chi_{i}=\chi_{i}\left(\mathcal{T}_{\lambda}\right)$. In our models, the mass abundances $\chi_{i}$ may change for two reasons. Firstly we are dealing with O- and/or C-stars. Secondly for the same type of star (either O- or C-stars) the opacities and the relative abundances of the grains can change with the optical depth (see Sect. 4). Since $k_{\lambda}$ and $\mathcal{T}_{\lambda}$ are each other interwoven, an iterative procedure is required ${ }^{1}$.

We also need the cross sections of the radiation-dust interactions. The cross section for a single dimension of the grains $a$ is $\sigma_{\mathrm{g}_{i}}(a)=\pi a^{2} Q_{\text {ext }}(i)$, where $Q_{\text {ext }}(i)$ are the extinction coefficients. The total cross section $\sigma_{\mathrm{g}_{i}}$ can be obtained integrating the cross section for a single dimension over a given distribution of the dimensions of the grains (see Sect. 5 for a discussion about the choice we have made for the distribution). The absorption and scattering coefficients, $Q_{\text {abs }}(i)$ and $Q_{\text {sca }}(i)$ respectively, can be calculated at any given wavelength with

1 The radiative transfer code "DUSTY" refers the optical depth $\mathcal{T}_{\lambda}$ at a wavelength of reference. Similarly to Suh $(1999,2000,2002)$, we assume as reference wavelength $\lambda=10 \mu \mathrm{m}$. This implies that the term $k_{\lambda}$ in the optical depth equation has to be evaluated at this wavelength, once the dust composition is fixed. 
the aid of the Mie theory ${ }^{2}$. The parameters to be specified are: the optical constants of the material composing the dust grains, the dimension and shape of the grains that are usually approximated to a sphere. Basing on this, the absorption and scattering coefficients, $Q_{\text {abs }}$ and $Q_{\text {sca }}$, can be derived from the complex dielectric function, expressed by the real $n(i)$ and the imaginary part $k(i)$ of the complex index of refraction (Bohren \& Huffman 1983; Draine \& Lee 1984).

The expansion velocity of the matter can be derived from the Vassiliadis \& Wood (1993) relationship correlating the expansion velocity to the pulsational period

$v_{\text {exp }}\left[\mathrm{km} \mathrm{s}^{-1}\right]=-13.5+0.056 P$

with the condition that the velocity is higher than $3 \mathrm{~km} \mathrm{~s}^{-1}$ (Vassiliadis \& Wood 1993). The pulsation period $P$ depends on the pulsation mode of the star.

A simple relation between period, mass and radius (Eq. (4) in Vassiliadis \& Wood 1993) is obtained supposing that AGB variable stars pulsate into the fundamental mode:

$\log P=-2.07+1.94 \log \left(\frac{R}{R_{\odot}}\right)-0.9\left(\frac{M}{M_{\odot}}\right)$

where the stellar radius $R$ and mass $M$ are expressed in solar units.

Another variable that appears in Eq. (12) is the dust-togas ratio $\delta$. We need to relate this ratio to the stellar parameters. Habing et al. (1994) studying the dependence of the gas outflow velocity $v_{\exp }$ at large distances from the star, found that $v_{\exp }$ depends on three parameters, that is the stellar luminosity $L$, the mass-loss rate $\dot{M}$, and the dust-to-gas ratio $\delta$. The observational data indicate that $v_{\text {exp }}$ increases at increasing any one of these parameters as also confirmed by the models of Habing et al. (1994) who also suggested a plausible correlation between $v_{\exp }$ and the dimensions $a$ of the grains. Therefore, $v_{\exp }=v_{\exp }(L, \delta, \dot{M}, a)$ is a function of four variables. However, $v_{\text {exp }}$ is found to depend only weakly on the mass-loss rate $\dot{M}$ except when $\dot{M}$ is small and the same weak dependence holds good for the grain dimension $a$. See Habing et al. (1994) for all details. Since our aim is to include the effects of the dust shell around AGB stars on the spectra of SSPs, stars with a thick shell and high mass-loss rates give the dominant contribution to IR spectrum of the SSP. Therefore, we adopt here the relation of Habing et al. (1994) for high mass-loss rates, and neglect the dependence of $v_{\text {exp }}$ on $\dot{M}$ and $a$. The expression for $v_{\text {exp }}$ in presence of high mass-loss rates is

$v_{\text {exp }} \propto L_{*}^{0.3} \delta^{0.5}$.

Following Bressan et al. (1998), who made use of the results by Habing et al. (1994), we recast $v_{\text {exp }}$ as

$v_{\text {exp }} \simeq 15\left[\mathrm{~km} \mathrm{~s}^{-1}\right]\left(\frac{L}{10^{4} L_{\odot}}\right)^{0.3}\left(\frac{\delta}{\delta_{\mathrm{AGB}}}\right)^{0.5}$

2 The Mie theory, developed in 1908 by Gustav Mie to understand the colors generated by gold particles suspended in water, provides a formal solution for the interaction of spherical and homogeneous small particles with the electromagnetic radiation (Bohren \& Huffman 1983). and eventually invert it to estimate $\delta$. The factor $\delta_{\mathrm{AGB}}$ depends on the kind of star under consideration. For the O-stars we use $\delta_{\mathrm{AGB}}=0.01$ as in Bressan et al. (1998) and Suh (1999), whereas for the C-rich stars we adopt the value $\delta_{\mathrm{AGB}}=0.0025$ computed by Blanco et al. (1998).

\section{Mass-loss rates from AGB stars}

The rate of mass-loss along the AGB is a key parameter for the evolution of the stars in this phase because it affects their lifetime and luminosity.

Despite the great effort devoted to clarify the role of massloss on the evolution of AGB stars, the mechanism of mass-loss itself is not completely clear. The determinations of the massloss rate from IR and radio data, are affected by significant uncertainties, due to the poorly known distances, dust-to-gas ratio, and expansion velocity of the sources.

There are two nearly direct observational evidence for mass-loss: the continuum IR emission in excess to what we expect from a star with the typical effective temperature of an AGB, and the molecular rotational lines detected in emission. The IR emission observed is characteristic of a dusty shell that absorbs the light of the star and then emits it in the IR and radio range. The molecular lines come from the gas surrounding the star: their widths and time variations prove that the gas is flowing away from the star.

It is now widely accepted and supported by hydrodynamical models that large amplitude pulsations are essential for accelerating the mass outflow from the stellar surface of AGB stars until the gas becomes cool enough that heavy elements can condense into dust grains. The dust grains in turn absorb and scatter the radiation transferring by collisions energy and momentum from the stellar radiation field to the gas so that the flow velocity may exceed the escape velocity (Gilman 1972). Mass-loss grows with time until the so-called superwind regime sets in, which quickly turns the star into a planetary nebula by stripping away all the envelope and leaving a bare core that evolves to high temperatures.

Vassiliadis \& Wood (1993) represented the above situation with suitable analytical fits in which $\dot{M}$ exponentially grows with the luminosity up to the formation of the planetary nebula. Following the formalism of Vassiliadis \& Wood (1993) we have adopted the relations below to express the rate of massloss prior and during the super-wind regime. The transition occurs at a period of about 500 days. Furthermore, once the superwind regime has started the stellar envelope gets so rich in dust that the star is no longer observable in the visible range of the spectrum.

Prior to super-wind, the mass-loss rate is expressed by

$\log \dot{M}=-11.4+0.0123 P$

where $\dot{M}$ is in $M_{\odot} \mathrm{yr}^{-1}$ and the period is in days. During the super-wind regime $\dot{M}$ is assumed to be given by

$\dot{M}=\frac{1}{c v}\left(\frac{L}{L_{\odot}}\right)$

which describes a wind driven by radiation pressure (Ivezic \& Elitzur 1994). 
As already pointed out by Vassiliadis \& Wood (1993) long period variables detected in the optical are found up to periods of 750 days (top upper part of the AGB where the stellar mass is $\sim 5 M_{\odot}$ ). In order to take this into account, Vassiliadis \& Wood (1993) slightly changed the expression for the mass-loss rate of Eq. (21) according to

$\log \dot{M}=-11.4+0.0125 \times\left[P-100\left(\frac{M}{M_{\odot}}-2.5\right)\right]$

for stars with masses $M>2.5 M_{\odot}$. Once again $\dot{M}$ is in $M_{\odot} \mathrm{yr}^{-1}$, and the period is in days. The Eqs. (21)-(23) completely describe our mass-loss prescriptions for AGB stars.

\section{Formation and properties of the dusty circumstellar envelopes}

O-rich stars. O-rich AGB stars of M spectral type show two typical features at $10 \mu \mathrm{m}$ and $18 \mu \mathrm{m}$ either in absorption or in emission depending on the optical depth of the surrounding envelope. These features are usually attributed to stretching and bending modes of $\mathrm{Si}-\mathrm{O}$ bonds and $\mathrm{O}-\mathrm{Si}-\mathrm{O}$ groups and clearly probe the existence of silicate grains in the shell of matter around the star.

Among AGB stars, the $\mathrm{OH} / \mathrm{IR}$ stars are generally thought to represent the final evolutionary stage of an O-rich object, just before it evolves quickly into a planetary nebula. Conversely, Mira variables of $\mathrm{M}$ spectral type are thought to correspond to early or advanced AGB evolutionary stages of oxygenrich stars. An oxygen-rich star is characterized by the ratio $\mathrm{C} / \mathrm{O}<1$. Because of the strong triple bond between $\mathrm{O}$ and $\mathrm{C}$ in the carbon monoxide, it is believed that all $\mathrm{C}$ will be blocked into $\mathrm{CO}$ molecules and no $\mathrm{C}$ is available to the formation of dust grain with other elemental species of low abundance. In contrast, the fraction of $\mathrm{O}$ not engaged in $\mathrm{CO}$ reacts with other elements such as $\mathrm{Mg}$ and $\mathrm{Si}$ and forms various types of compounds. Therefore, an O-rich star is characterized by the presence of $\mathrm{O}$ and compounds like $\mathrm{MgO}$, silicates and $\mathrm{H}_{2} \mathrm{O}$. Eventually, these molecules can bind together to produce grains of silicates.

The newly formed dust grains leave the envelope of the AGB stars and disperse into the interstellar medium, where they can be strongly modified by chemical and physical processes, such as collisions, interaction with energetic photons, accretion and destruction of the mantle, and so forth (see $\mathrm{Li}$ \& Mayo Greenberg 2002, for a recent review and referencing of the subject). Therefore the opacities of dust silicates in AGB stars are probably different from those derived from laboratory measurements of terrestrial or meteoritic material. Furthermore, the classical opacities of silicates of the diffuse interstellar medium cannot be used to model the dusty envelopes of AGB stars (Ossenkopf et al. 1992).

There are two final points to consider: i.e. the consistency of the opacities in use with the Kramers-Kronig dispersion relations (see e.g. Landau \& Lifshitz 1960) which imply that the real and imaginary parts of the complex dielectric function $\epsilon(\omega)=\epsilon_{1}+\mathrm{i} \epsilon_{2}$ are not independent, see also Bohren \& Huffman (1983), and the consistency of the theoretical results with the observational ones. In our case, the spectra obtained from radiative transfer models of AGB stars surrounded by dusty shells must agree with the IR observations of these stars. For more details on this subject see Draine \& Lee (1984).

As already pointed out by Suh (1999), the dust opacities are often adjusted in such a way that observations (spectra) are reproduced, however, without checking for the Kramers-Kronig dispersion relation at the same time (Volk \& Kwok 1988; Griffin 1993; Suh \& Jones 1997). In some cases (Ossenkopf et al. 1992) the physical consistency of the input opacity is secured, but no comparison between observational data and theoretical predictions is made. A rare exception is Suh (1999) who presents optical constants that satisfy both the Kramers-Kronig relation and properties of O-rich AGB stars better than in previous studies. In addition to this, Suh (1999) gives two estimates for the silicate opacities, one for the warm grains (obtained reproducing the emission feature at $10 \mu \mathrm{m}$ of $\mathrm{OH} / \mathrm{IR}$ stars) and one for cold grains (obtained reproducing the absorption feature at $10 \mu \mathrm{m}$ always of $\mathrm{OH} / \mathrm{IR}$ stars). The variation of the silicate opacity with the observational constraint to be reproduced can be understood by considering that AGB stars can be surrounded by thin or thick shells and that the grain temperature is likely to be cooler in thick shells than in thin shells. A simple argument supporting this possibility is that in AGB stars surrounded by a thin shell of matter a large part of the dust will be at high temperatures, whereas in AGB stars with a thick circumstellar shell, dust will be at lower temperatures. Basing on this, Suh (1999), argues that different kinds of grain (opacities) are required at varying optical depth of the shell: cold silicates are suited to thick shells with the $10 \mu \mathrm{m}$ feature in absorption, whereas warm silicates are more appropriate to thin shells with the $10 \mu \mathrm{m}$ feature in emission. The largest difference between the opacities of the two species occurs at $\lambda>13 \mu \mathrm{m}$ (see the top panel of Fig. 1) and according to Suh (1999) the transition between the two physical situations occurs at the optical depth $\mathcal{T}_{10}=3$ and $\lambda=10 \mu \mathrm{m}$. The same values are adopted here.

The mid-infrared spectra of oxygen-rich circumstellar envelopes are dominated by the silicate bands at about 10 and $19 \mu \mathrm{m}$ and so much less attention has been paid to the other IR spectral regions until the advent of IRAS and ISO in particular. However, in many IRAS LRS spectra of Mira variables (Sloan et al. 1996) an emission band at about $13 \mu \mathrm{m}$ has been detected that could be due to aluminum oxide (Begemann et al. 1997), but its origin is still a matter of vivid debate (Posch et al. 1999). Other weak silicate features around 10 and $18 \mu \mathrm{m}$ have been detected by the ISO-SWS observations which can be attributed to the presence of oxide particles. In many AGB stars with high mass-loss rates, ISO high resolution observations revealed the presence of prominent bands of crystalline silicates, for instance enstatite $\left(\mathrm{MgSiO}_{3}\right)$ and forsterite $\left(\mathrm{Mg}_{2} \mathrm{SiO}_{4}\right)$ (Waters et al. 1996; Waters \& Molster 1999).

All these features spurred the interest toward the composition of circumstellar dusty shells and grain formation theories (Gail \& Sedlmayr 1999). Suh (2002) presented a dust model for the envelopes of O-rich stars in which not only amorphous silicates but also crystalline silicates are included. The adopted opacity functions are those of Jaeger et al. (1998) (the bottom panel of Fig. 1). The key parameter controlling an envelope 


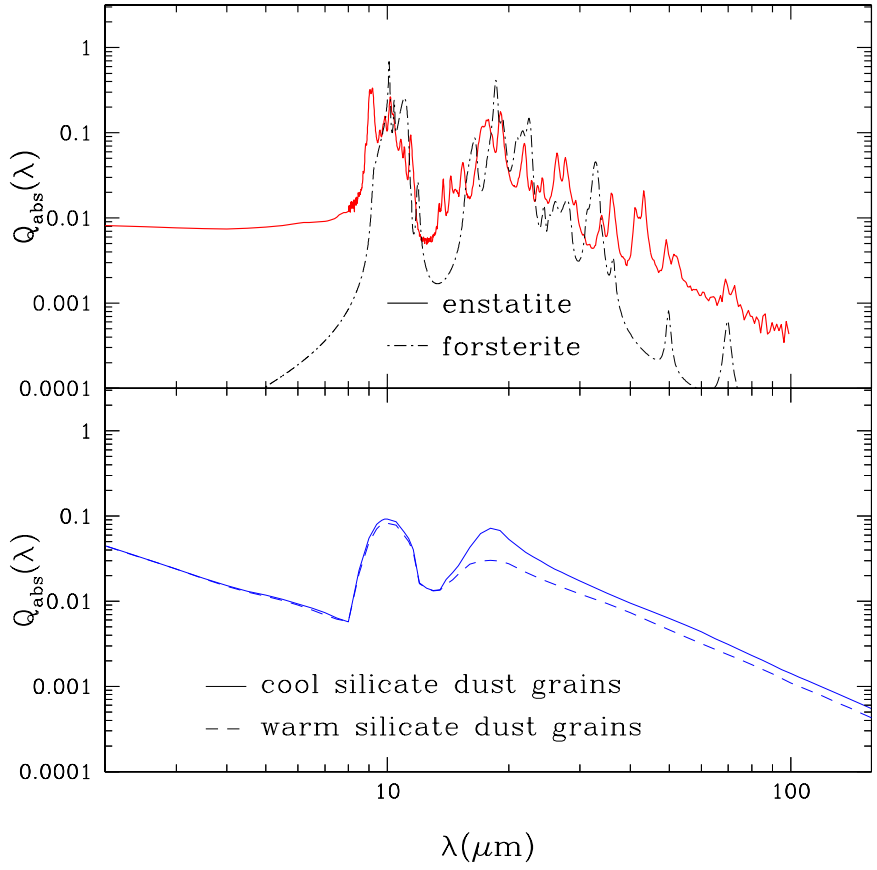

Fig. 1. Upper panel: extinction coefficients for two crystalline silicates i.e. forsterite from Jaeger (2002, private communication) and enstatite from Jaeger et al. (1998). Bottom panel: extinction coefficients for cool and warm silicates taken from Suh (2002).

composed by amorphous silicates and crystalline silicates is the so-called crystallinity parameter $\alpha$, that is the ratio between total mass of crystalline silicates to the total mass of silicates. Suh (2002), examining various levels of crystallinity, found that for the same value of $\alpha$, crystalline silicate features are stronger for optical depths smaller than 5 and weaker for optical depths larger than 10 (always referred to the optical depth at $10 \mu \mathrm{m}$ ). A high crystallinity is required to produce strong features in models with high optical depth.

The intensity of the crystalline silicate features is likely to correlate with the mass-loss rate from AGB stars. As a matter of fact these features are stronger in OH/IR stars with high mass-loss rates (Sylvester et al. 1999). Because the observations show that the same features are not present in stars with low mass-loss rates, one may perhaps infer that these stars are also deficient of crystalline silicates in their envelopes (Suh 2002). The problem, however, needs deeper analysis because Suh (2002) and Kemper et al. (2001) find contrasting results. Kemper et al. (2001) even including the presence of crystalline silicates in the dusty envelopes, fail indeed to reproduce the features in question.

Following Suh (2002), we adopt here the crystallinity parameter $\alpha=0.1$ for stars with low mass-loss rates and moderately optically thick shells of matter $\left(\mathcal{T}_{10}<15\right)$, whereas for $\mathrm{OH} / \mathrm{IR}$ stars with high mass-loss rates and optically thick shells $\left(\mathcal{T}_{10}>15\right)$ we prefer the value $\alpha=0.2$. It is worth recalling that $\alpha=0.2$ is fully sufficient to reproduce the prominent crystalline features shown by $\mathrm{OH} / \mathrm{IR}$ spectra. Finally, in all the models the relative contents of enstatite $\left(\mathrm{MgSiO}_{3}\right)$ and

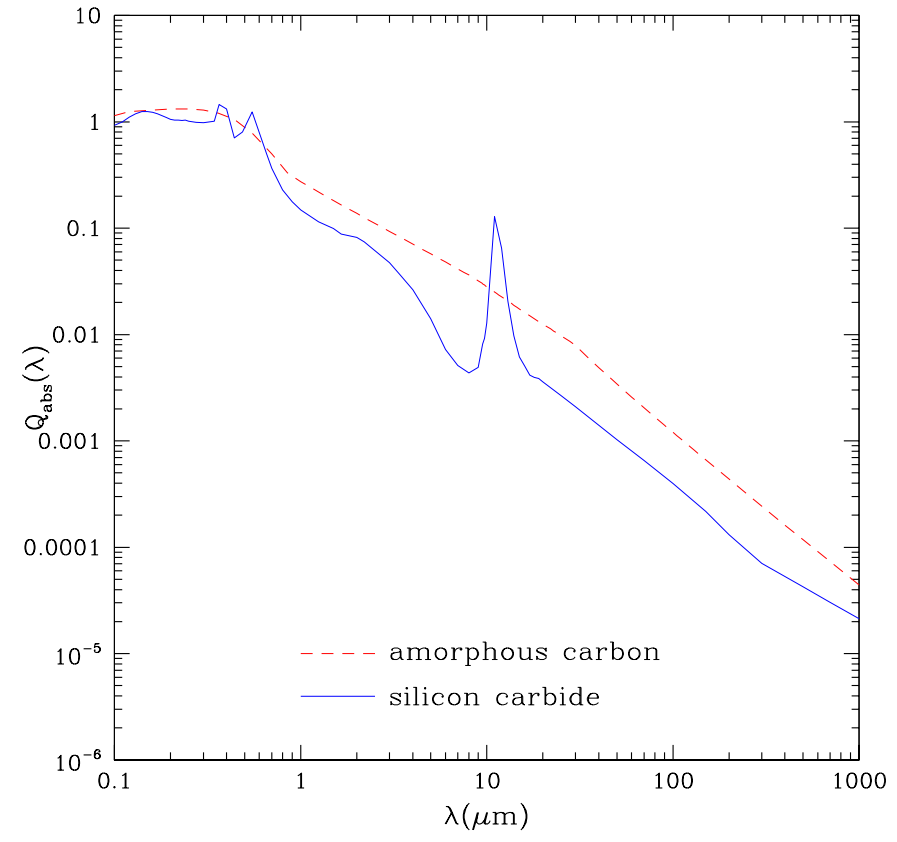

Fig. 2. Absorption coefficients for amorphous carbon (dashed line) from Suh (2000) and silicon carbide (solid line) from Pégourié (1988).

forsterite $\left(\mathrm{Mg}_{2} \mathrm{SiO}_{4}\right)$ are the same as in Suh (2002), i.e. we adopt the same relative contribution for the two components.

C-rich stars. C-stars are the evolutionary descendants of Mira variables. Through continuous dredge-up of carbon into the envelope during the thermally pulsing AGB phase, these stars eventually reach a carbon abundance in the outer layers larger than that of oxygen $(\mathrm{C} / \mathrm{O}>1)$. When this occurs the formation of O-rich dust ceases and it is replaced by that with C-rich compounds: the C-star phase begins. Therein after, the continuous formation of C-rich dust makes the envelopes of these stars more and more optically thick. By loosing mass at very high rates, they get enshrouded by thick envelopes that absorb and scatter the UV-optical radiation into the IR and radio range.

Almost all these stars show an emission feature at $11.3 \mu \mathrm{m}$, due to silicon carbide ( $\mathrm{SiC}$ ), whose presence was predicted by Gilman (1969) and observationally confirmed by Hackwell (1972). Many infrared observations have brought into evidence and confirmed the presence of several types of dust grains in C-rich AGB stars: amorphous carbon (AMC), silicon carbide, and magnesium sulphide $(\mathrm{MgS})$ to mention the three dominant types. Suh $(1999,2000)$ has derived new opacities for the AMC, that are consistent with Kramers-Kronig dispersion relations, and has also reproduced the observational data by means of suitable models of the dusty shells around AGB stars (see Fig. 2). The models are based on the new opacities and a complete treatment of the radiative transfer, thus much improving upon previous studies (Blanco et al. 1998; Groenewegen et al. 1998). The Suh $(1999,2000)$ models are characterized by two components, SiC and AMC (for typical models of carbon stars, see for example Lorenz-Martins et al. (2001) and references therein). The $30 \mu \mathrm{m} \mathrm{MgS}$ feature is observed in a wide range of sources, going from low mass-loss carbon stars 
to planetary nebulae (Hony et al. 2002). Because it is observed in C stars of large luminosity it is expected to significantly contribute to the integrated spectrum in the $30 \mu \mathrm{m}$ range. However, as the optical constants of $\mathrm{MgS}$ are not measured for a sufficiently wide range of wavelengths (Suh 2000; Hony et al. 2002) the presence of $\mathrm{MgS}$ is neglected here.

The grains of silicon carbide can be separated in two groups, hexagonal or rhombohedral $\alpha \mathrm{SiC}$ and cubic $\beta \mathrm{SiC}$. Many authors (Groenewegen 1995; Speck et al. 1997; Blanco et al. 1998) argue that in order to fit the IR spectra of C-stars, $\alpha \mathrm{SiC}$ is required sometimes together with $\beta \mathrm{SiC}$. However, all studies of meteoritic $\mathrm{SiC}$ grains have revealed the presence of only $\beta \mathrm{SiC}$. Since it is thermodynamically unlikely that $\alpha \mathrm{SiC}$ transforms itself into $\beta \mathrm{SiC}$ (Bernatowicz 1997), there seems to be a clear discrepancy between the $\alpha \mathrm{SiC}$ suggested by the AGB spectra and the $\beta \mathrm{SiC}$ indicated by the meteoritic data. As pointed out by Speck et al. (1999) the discrepancy could be due to inadequate $\mathrm{KBr}$ corrections ${ }^{3}$ to the laboratory spectra so that a plausible way out can be found.

$\mathrm{AMC}$ and $\mathrm{SiC}$ affect the spectrum in a different fashion: while the effects of AMC propagate over the whole spectrum, those of $\mathrm{SiC}$ are limited to the $11 \mu \mathrm{m}$ feature as observationally indicated. Lorenz-Martins \& Lefevre (1994) and Groenewegen (1995) suggest that the ratio $\mathrm{SiC}$ to AMC decreases at increasing optical depth of the dusty envelope. This is also confirmed by the models of Suh (1999). Therefore, following Suh (1999) prescription, we assume here that the chemical composition of the dust changes with the optical depth of circumstellar shell. For optically thin dust shells $\left(\mathcal{T}_{10} \leq 0.15\right)$, the strong $11 \mu \mathrm{m}$ feature requires about $20 \%$ of $\mathrm{SiC}$ dust grains to fit the observational data for AGB; for dust shells with intermediate optical depth $\left(0.15 \leq \mathcal{T}_{10} \leq 0.8\right)$ about $10 \% \mathrm{SiC}$ dust grains are needed, whereas for shells with larger optical depths, in which the $11 \mu \mathrm{m}$ feature is either much weaker or missing at all, no SiC is required. Following Suh (1999), who used the optical constants of $\alpha \mathrm{SiC}$ by Pégourié (1988) to calculate the opacity for $\mathrm{SiC}$ and reproduce the spectra of C-stars similarly, we adopt here the same data (see Fig. 2).

\section{Parameters of the shell model}

An important parameter of the dusty shell models is the distribution of the grain dimensions. Nowadays many recipes have been proposed to reproduce the properties of the dust grains: they go from the classical power-law (Mathis et al. 1977) to a log-normal distribution of Weingartner \& Draine (2001). In this study we adopt the simple distribution expressed by the Dirac delta function

$n(a)=\delta\left(a-a_{0}\right)$

\footnotetext{
${ }^{3}$ Dorschner et al. (1978) have studied the effects of the a dispersive medium made of potassium bromide $(\mathrm{KBr})$ on the spectra of small quantities of silicates with the grain size. They find a shift of the spectral features emitted by the grains using either a $\mathrm{KBr}$-matrix or a $\mathrm{KBr}$-pellet in which the small grains are embedded. They explain the shift as due to an effect caused by the $\mathrm{KBr}$-matrix that needs to be corrected before using the spectra obtained with the KBr-pellet. See Speck et al. (1999) for more details about the problem of the $\mathrm{KBr}$ correction.
}

with $a_{0}=0.1 \mu \mathrm{m}$, where $a$ is the dimension of the grains. Despite its simplicity, this type of distribution is fully adequate to our purposes and it has already been widely used to model the dusty shells of AGB stars (Hashimoto 1995; Ivezic \& Elitzur 1995; van der Veen et al. 1995; Suh \& Jones 1997; Suh 1997, 1999; van Loon et al. 1999; Suh 2000, 2002). Support to this kind of approximation is by Bagnulo et al. (1995) who find that a delta-function fits the spectrum of IRC+10216 much better than a power law.

Since different types of grains are present in the dust mixture of the circumstellar shell, this could imply different values of the grain temperature at the same distance from the central source. As a consequence of it, the condensation temperature at inner boundary of the shell could vary with the type of grain. In the case of shells of the C-rich stars, for which we use a mixture of $\mathrm{AMC}$ and $\mathrm{SiC}, T_{\mathrm{d}}$ is assumed equal to $1000 \mathrm{~K}$ for both types of grain: in reality $\mathrm{AMC}$ and $\mathrm{SiC}$ should have their own temperature and temperature profile across the shell. This of course would add additional parameters to the problem, i.e. two condensation temperatures and two dust-to-gas ratios, thus rendering the whole problem much more complicated and beyond the technical capability of the code DUSTY we are using (Nenkova et al. 1999). The code indeed treats a mixture of grains using a pseudo-grain whose optical properties are the average of those of individual species and of course deals with a single value of $T_{\mathrm{d}}$. Fortunately, in the case of C-rich stars, the abundance of $\mathrm{SiC}$ is very low so that the approximation to a single value of $T_{\mathrm{d}}$ is physically acceptable. By the same token, we may also assume the same value of $T_{\mathrm{d}}=1000 \mathrm{~K}$ for this type of shell. As first noticed by Suh (1999), if in an expanding stellar envelope dust grains condense in amorphous form and then get a crystalline structure on a short time scale, it is likely that two different types of grain have the same temperature distribution.

Another important parameter is the density profile of the grains across the shell. Many different density laws have been suggested: the simple power-law $\rho \propto r^{-x}$ is a popular choice even if the exponent $x$ varies from author to author. The law $\rho \propto$ $r^{-2}$ is often adopted. Equally good alternatives are $\rho \propto r^{-1}$ or $\rho \propto r^{-3}$. The latter seems more suited to describe the case of O-stars with thick envelopes. Furthermore, models with radiation pressure or models with pulsations and shocks pre$\operatorname{dict} \rho \propto r^{-2}$. Finally, a region of finite thickness with enhanced density is added to the distribution $\rho \propto r^{-2}$ to somehow describe stars that are in the super-wind phase (Suh \& Jones 1997; Suh 1997). In this study we simply adopt the power-law $\rho \propto r^{-2}$ as in the series of papers by $\operatorname{Suh}(1999,2000,2002)$ from which we also take the opacities of the dust grains.

However, using different power-laws for the distribution of the matter in the shell implies that the same overall optical depth is reached at different radii. In order to evaluate the uncertainty on the optical depth $\mathcal{T}_{\lambda}$ caused by using different density profiles across the shell. We start from Eq. (4), insert the relation $\rho_{\mathrm{d}}(r)=\delta \rho(r)$ where $\delta$ is a constant, suppose for the sake of simplicity that the product $k_{\lambda}(r) \delta$ does not depend on $r$, and finally insert the generic radial dependence for the density $\rho_{0}\left(r / r_{0}\right)^{-n}$ (where $\rho_{0}$ and $r_{0}$ are suitable scale factors). 
The optical depth down to a generic radius $r$ is

$$
\begin{aligned}
\mathcal{T}_{\lambda}(r) & =\left\langle k_{\lambda}(r) \delta\right\rangle \int_{r_{\mathrm{in}}}^{r} \rho(r) \mathrm{d} r \\
& =\left\langle k_{\lambda}(r) \delta\right\rangle \rho_{0} r_{0}^{n} \int_{r_{\mathrm{in}}}^{r} r^{-n} \mathrm{~d} r .
\end{aligned}
$$

At any given radius $r$ the variation caused by using different power-laws is

$\Delta\left(r_{0}^{n} \int_{r_{\text {in }}}^{r} r^{-n} \mathrm{~d} r\right)$

Let us indicate with $\mathcal{I}_{1}, \mathcal{I}_{2}$ and $\mathcal{I}_{3}$ the three integrals for three typical power-law exponents $n=1,2$ and 3, respectively, pose $r=\alpha r_{\text {in }}$, and finally assume the scale length $r_{0}$ to be the inner radius of the shell $r_{\text {in }}$. We immediately get

$\mathcal{I}_{1}=r_{\text {in }} \ln \alpha \quad \mathcal{I}_{2} \simeq r_{\text {in }}\left(1-\frac{1}{\alpha}\right) \quad \mathcal{I}_{3} \simeq 0.5 r_{\text {in }}\left(1-\frac{1}{\alpha^{2}}\right)$.

The factors multiplying $r_{\text {in }}$ give an idea of the differences due to the power-law exponent $n$. The net consequence of different mass density profiles is that the more matter is stored at large radii and hence lower temperatures the flatter is the spectrum and vice-versa (see Ivezic \& Elitzur 1997, for more details).

\section{Uncertainties of the shell model}

In this section, we try to discuss the uncertainties arising from the two main components of the adopted model for the dust shell.

Optical depth. Differentiating Eq. (12) we get

$\frac{\Delta \mathcal{T}_{\lambda}}{\mathcal{T}_{\lambda}}=\frac{\Delta \delta}{\delta}+\frac{\Delta \dot{M}}{\dot{M}}+\frac{\Delta k_{\lambda}}{k_{\lambda}}+\frac{\Delta v_{\exp }}{v_{\text {exp }}}+\frac{\Delta r_{\text {in }}}{r_{\text {in }}}$.

In the following we try to estimate the various terms composing this relation. If we look at Eq. (20), this one well represents the link between luminosity of the star $L_{*}$, outflow velocity $v_{\exp }$ and dust-to-gas ratio $\delta$, because (Habing et al. 1994) if $L_{*}$ or $\delta$ increase, also $v_{\text {exp }}$ increases, but the exponents that rule the relation of proportionality are uncertain. We adopt the dependence proposed by Habing et al. (1994), but for example van Loon (2000) proposed a slightly different choice for the $L$ dependence. If we write Eq. (20) as $v_{\exp } \propto L_{*}^{\alpha} \delta^{\beta}$ we get

$\frac{\Delta \delta}{\delta}=\frac{1}{\beta} \frac{\Delta v_{\exp }}{v_{\exp }}+\frac{\alpha}{\beta} \frac{\Delta L}{L}$

where $\alpha$ and $\beta$ are 2 and 0.6 in our model. The errors $\Delta v_{\text {exp }}$ and $\Delta L$ are small, because Eqs. (17) and (18) are sufficiently good and so, if we consider a large spread of $\alpha$ and $\beta$ and typical values for the expansion velocities and luminosities of AGB stars, we get $\Delta \delta / \delta \simeq 1$ in the worse cases.

The uncertainty on the rate of mass-loss can be estimated from Eq. (21) prior to super wind and Eq. (22) during the superwind. Differentiating Eq. (21) we obtain

$\frac{\Delta \dot{M}}{\dot{M}}=\frac{1}{\log _{10} e} 0.0123 \Delta P \simeq 0.034 \Delta P$ which for the error on the period coming from (18) yields that $\Delta \dot{M} / \dot{M}$ is negligible. The uncertainty on Eq. (22) is simply

$\frac{\Delta \dot{M}}{\dot{M}}=\frac{\Delta L}{L}+\frac{\Delta v_{\mathrm{exp}}}{v_{\exp }}$

which is also negligible.

Estimating the term $\Delta k_{\lambda} / k_{\lambda}$ is a cumbersome affair because $k_{\lambda}$ depends on the mass abundances $\chi_{i}$ of the grains, which in turn are functions of the optical depth $\mathcal{T}_{\lambda}$ and of the cross sections $\sigma_{\mathrm{g}_{i}}$ s of the dust-radiation interactions, which depend in its turn on the grain dimensions $a$ and on the extinction coefficients $Q_{\text {ext }}(i)$. No simple way of checking the uncertainty can be found, but referring to the original sources of data for extinction properties.

As already mentioned, the uncertainty on the expansion velocity $v_{\exp }$ is simply estimated from Eq. (17), which yield a negligible $\Delta v_{\text {exp }} / v_{\text {exp }}$.

Finally, the uncertainty on the inner radius of the shell can be derived from differentiating Eq. (14)

$\frac{\Delta r_{\text {in }}}{r_{\text {in }}}=2 \frac{\Delta T_{\mathrm{d}}}{T_{\mathrm{d}}}+2 \frac{\Delta L}{L}$.

For $T_{\mathrm{d}}$ we have many different values in literature: varying it in the realistic range from 1000 to $1500 \mathrm{~K}$ and taking the typical luminosity for AGB stars we estimate $\Delta r_{\text {in }} / r_{\text {in }} \simeq 1$. We can finally conclude that the terms $\Delta r_{\text {in }} / r_{\text {in }}$ and $\Delta \delta / \delta$ are the main source of error in the calculation of the optical depth $\mathcal{T}_{\lambda}$. For individual stars the error may be as large as $\Delta \mathcal{T}_{\lambda} / \mathcal{T}_{\lambda} \simeq 2$ which is acceptable for the purposes of our study. It will be smaller as soon as better determinations of the relationship $v_{\exp }\left(L_{*}, \delta\right)$ will be available.

Chemistry. Although the list compounds we have considered in modelling the chemical composition of the dust shells is certainly incomplete, an effort has been made to include as many molecules as possible. Many other compounds are of course possible and should be included in the list, but we need deeper studies able to discriminate for which AGB stars these compounds have to be included. However, compared to similar studies on the same topic, e.g. Bressan et al. (1998), our treatment is a significant step forward. To conclude we are confident that our description of the shell composition is adequate to the present aims. No doubt that this aspect can be improved.

Final remarks. Even if the uncertainty is as large as $\Delta \mathcal{T}_{\lambda} / \mathcal{T}_{\lambda} \simeq 2$, the situation is, however, not as bad as it may seem, because the largest effect of the dust shell on reprocessing the radiation coming from the central star underneath is for the optically thick case, in which the exact value of the optical depth is less of a problem.

\section{Theoretical SSPs: Basic ingredients}

The monochromatic flux of an SSP of age $t$ and metallicity $Z$ at the wavelength $\lambda$ is defined as

$\operatorname{ssp}_{\lambda}(t, Z)=\int_{M_{\mathrm{L}}}^{M_{\mathrm{U}}(t)} f_{\lambda}(M, t, Z) \Phi(M) \mathrm{d} M$

where $f_{\lambda}(M, t, Z)$ is the monochromatic flux emitted by a star of mass $M$, age $t$, and metallicity $Z ; \Phi(M)$ is the initial mass 
function (IMF); $M_{\mathrm{L}}$ is the mass of the lowest mass star in the SSP whereas $M_{\mathrm{U}}(t)$ is the mass of the highest mass star still alive in the SSP of age $t$. For the IMF we adopt the Salpeter (1955) law expressed as

$\frac{\mathrm{d} N}{\mathrm{~d} M}=\Phi(M)=\mathcal{A} M^{-x}$

where $x=2.35$ and $\mathcal{A}$ is a normalization constant to be fixed by a suitable condition (SSPs for other choices of the IMF can be easily calculated).

Because the flux $\operatorname{ssp}_{\lambda}(t, Z)$ is calculated by integrating Eq. (31) along an isochrone, one has to know the luminosity, $T_{\text {eff }}$ and gravity of the stars of mass $M$, age $t$ and metallicity $Z$ lying on the isochrone. For any star of mass $M$, age $t$ and metallicity $Z$, the relationship between luminosity, $T_{\text {eff }}$, gravity with the age $t$ is derived from a library of stellar models, the flux $f_{\lambda}(M, t, Z)$ from a library of stellar spectra. Finally one has to adopt a prescription for the amount of mass lost by a star of mass $M$ in the course of its evolution: this could occur all over the evolutionary history such as in case of massive stars, or at the end of the RGB passing from the tip of the RGB to the $\mathrm{HB}$ or clump as appropriate, and during the AGB phase as in the case of low and intermediate mass stars. All details of the isochrone construction technique can be found in Bertelli et al. (1994).

\subsection{Libraries of stellar models and stellar spectra}

In our study we adopt the isochrones by Tantalo (1998) (anticipated in the data base for galaxy evolution models by Leitherer et al. 1996). The underlying stellar models are those of the Padova Library. They are shortly referred to as the Bertelli et al. (1994) stellar models (see references therein for more details). These stellar models are calculated with convective overshooting and are amply described by Bertelli et al. (1994) so that no detail is given here. In the present study only three chemical compositions are considered, namely $[Y=0.240, Z=0.004]$, [ $Y=0.250, Z=0.008],[Y=0.280, Z=0.02]$, for which the transition luminosities for AGB stars passing from the O-rich to the C-rich regime calculated by Marigo et al. (1999) are available (see below).

In the isochrones, all evolutionary phases from ZAMS to the end of the TP-AGB or C-ignition are included, as appropriate to the mass of the last living star of age $t$. The rate of mass-loss during the RGB stages of low mass stars is from Reimers (1975) with $\eta=0.45$. During the AGB phase the rate of mass-loss is according to Vassiliadis \& Wood (1993), as already described in Sect. 3. The initial masses of the stellar models go from 0.15 to $100 M_{\odot}$ and the ages of the isochrones go from 3 Myr to 20 Gyr.

The library of stellar spectra is from Lejeune et al. (1998), which stands on the Kurucz (1995) release of theoretical spectra, however with several important implementations. For $T_{\text {eff }}<3500 \mathrm{~K}$ the spectra of dwarf stars by Allard \& Hauschildt (1995) are included and for giant stars the spectra by Fluks et al. (1994) and Bessell et al. (1989, 1991) are considered. Following Bressan et al. (1994), for $T_{\text {eff }}>50000 \mathrm{~K}$, the library has been extended using black body spectra.

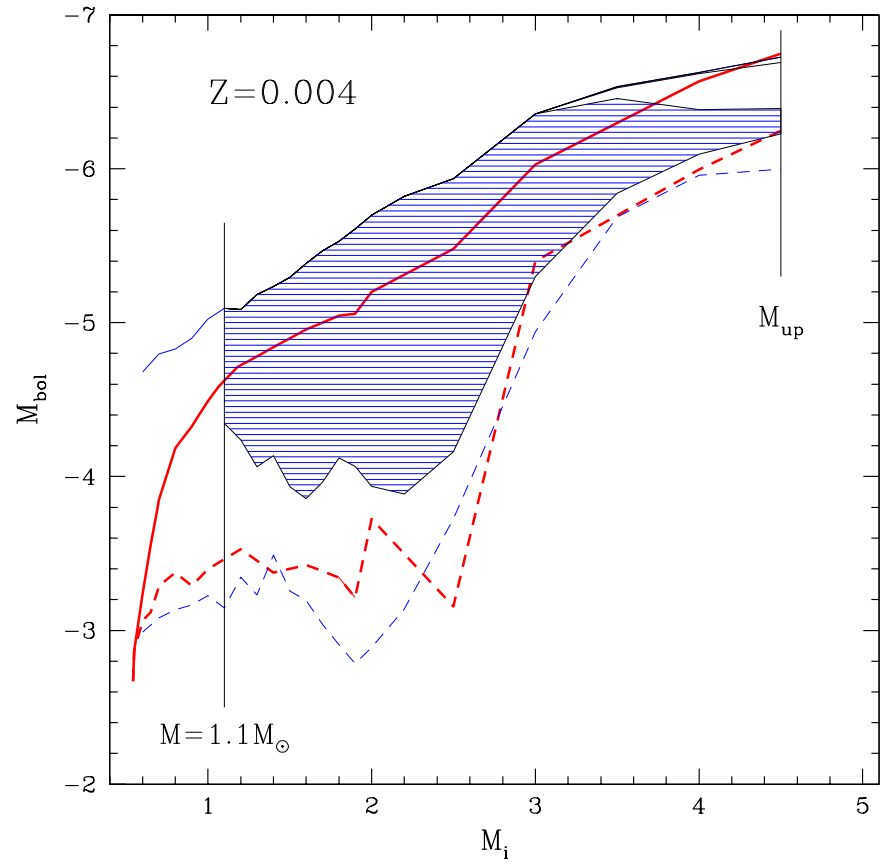

Fig. 3. The range of bolometric magnitudes spanned by AGB stars with $Z=0.004$ and the transition bolometric magnitudes from M- to $\mathrm{C}$-star as function of the initial mass. The dashed lines show the start of the AGB phase. The solid lines show the end of it. The shaded area is the luminosity interval in which $\mathrm{C}$-stars are formed. The thick lines (dashed and solid) are the models by Tantalo (1998) calculated with the Vassiliadis \& Wood (1993) prescription for mass-loss during the AGB phase. The thin lines (dashed and solid) show the same but for the models by Marigo et al. (1999). The region of C-stars is according to the Marigo et al. (1999) models. The two vertical lines show the minimum mass for the formation of $\mathrm{C}$-stars and the maximum mass $M_{\text {up }}$ for the occurrence of the AGB phase.

For ages younger than about 100 Myr the SSPs we are going to present will be equal to the old ones of Tantalo (1998), because no AGB stars can be formed, whereas for older ages the effect of the circumstellar envelopes in processing the radiation coming from the central star is taken into account.

\subsection{Transition luminosities of the AGB phases}

Owing to the different composition of the ejecta much affecting the chemistry and physics of the circumstellar shell, the transition from the oxygen-rich M-type stars $(\mathrm{C} / \mathrm{O}<1)$ to the carbon-rich $\mathrm{C}$-type objects $(\mathrm{C} / \mathrm{O}>1)$ deserves particular care.

In view of the discussion below, it is useful to summarize the evolution of AGB stars eventually becoming C-stars. The situation is illustrated in Figs. 3-5 for three different values of the metallicity, namely $Z=0.004, Z=0.008$ and $Z=0.02$ respectively. Each plot shows the bolometric magnitude of TPAGB stars as a function of their initial mass $M_{\mathrm{i}}$ from the beginning to the end of the AGB phase when the outer envelope is completely removed by mass-loss. The thick dashed and solid line bind the luminosity attained by the stellar models of the Padova Library (Bertelli et al. 1994) used by Tantalo (1998) assuming the prescription for mass-loss of Vassiliadis $\&$ Wood (1993). The thin dashed and solid lines show the same 


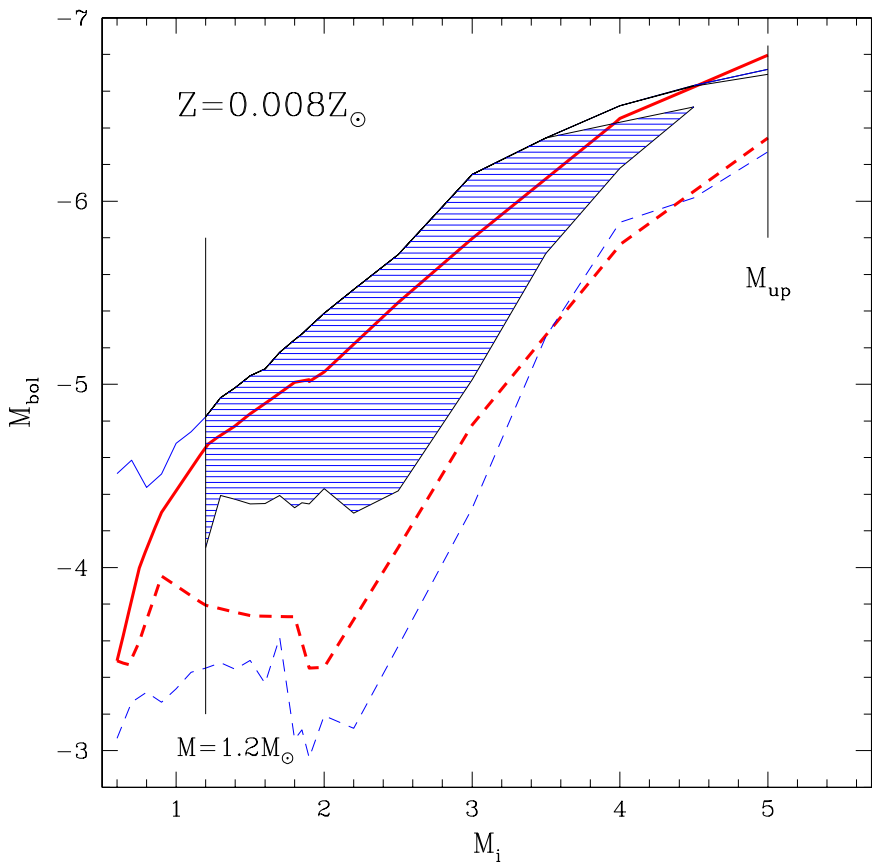

Fig. 4. The same as in Fig. 3 but for the metallicity $Z=0.008$.

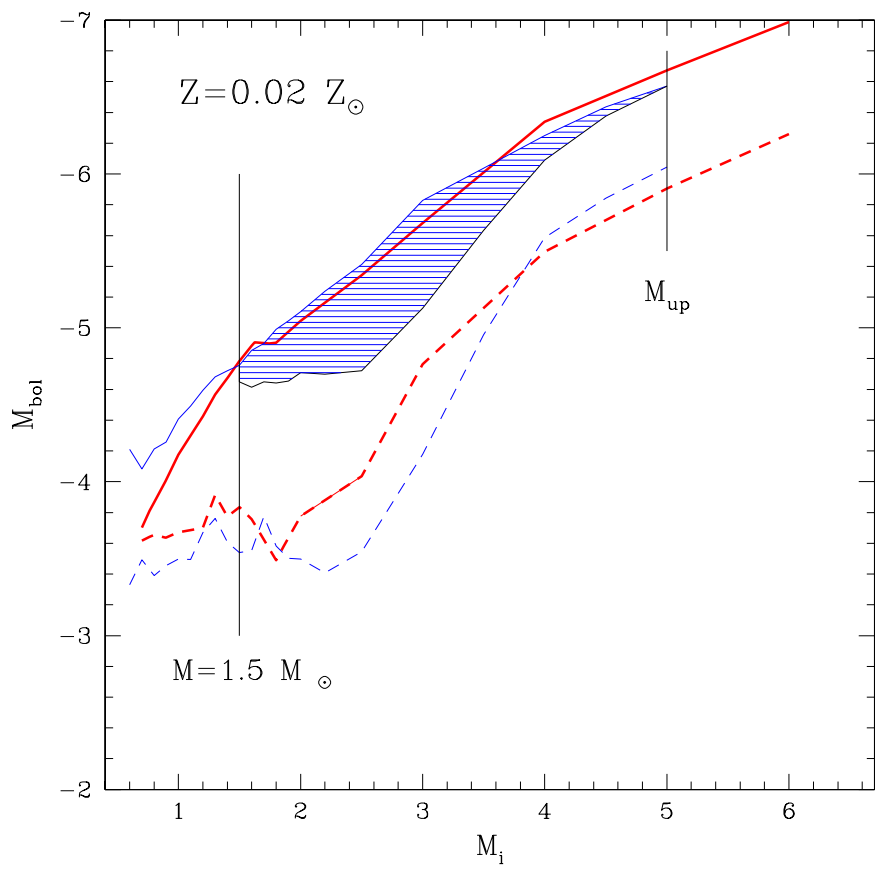

Fig. 5. The same as in Fig. 3 but for the metallicity $Z=0.02$.

according to the more recent models of TP-AGB stars by Marigo et al. (1999). The shaded areas correspond to the luminosity interval in which, according to Marigo et al. (1999), C-stars develop. Examining the three diagrams and the models by Marigo et al. (1999) in detail we note that:

(i) over the mass interval in which the AGB phase develops, the formation of C-stars does not occur for initial masses lower than a certain limit indicated by the vertical line (the limit mass increases at increasing metallicity). This is simply caused by the very early loss of the envelope before any change in the chemical composition of the outer layers may take place.
Therefore, in old stars only O-rich shells of dust with $\mathrm{C} / \mathrm{O}<1$ are possible. At larger initial masses, C-stars and C-rich dusty shells do occur over a wide range, which may extend up the so-called $M_{\text {up }}$, i.e the maximum value for the occurrence of the AGB phase, or slightly below this. In the range of the massive AGB, the occurrence of envelope burning may indeed delay the formation of a C-star, and in some cases may turn a C-star into a late O-rich star. More precisely, in this mass range a C-star is comprised between two stages, in which an AGB star appears as O-rich. The late O-rich phase is favored at low metallicities and disappears for $Z \gtrsim Z_{\odot}$.

(ii) As a consequence of the above trend, for the majority of C-stars (say up to $M_{\mathrm{i}} \lesssim 3.5 M_{\odot}$ ) the maximum luminosity just coincides with the end of the AGB phase, whereas for initial masses larger than $M_{\mathrm{i}} \gtrsim 3.5 M_{\odot}$ the maximum luminosity of C-stars may be fainter than the maximum luminosity of the AGB phase.

Given these premises, we need to incorporate the results by Marigo et al. (1999), which provide the transition luminosity into the isochrones (SSPs) by Tantalo (1998). The comparison of the initial, transition, and maximum luminosities in the two sets of models reveals that:

(a) For $Z=0.004$ the luminosity at which the TP-AGB phase begins is nearly the same for both Tantalo (1998) and Marigo et al. (1999), whereas the termination luminosity is lower in Tantalo (1998). Finally C-stars are formed for initial masses larger than about $1.1 M_{\odot}$. Owing to the lower termination luminosity, the proportion of C-stars with respect to that of the O-rich ones predicted by the Tantalo (1998) will be somewhat smaller compared to the value expected from Marigo et al. (1999).

(b) Similar considerations apply to the case with $Z=0.008$, the only difference being that no $\mathrm{C}$-stars can be formed for initial masses lower than about $1.2 M_{\odot}$. We note that the range of bolometric magnitude predicted by the models of Tantalo (1998) is narrower than that by Marigo et al. (1999), with lower maximum and higher initial luminosities for the AGB phase. This reduces the allowed range for both $\mathrm{M}$ - and C-stars. Nevertheless, to a first approximation the percentage of C-stars with respect to that of O-rich stars remains similar to that predicted by Marigo et al. (1999).

(c) For $Z=Z_{\odot}$, the initial and maximum luminosity of the TP-AGB phase in Tantalo (1998) and Marigo et al. (1999) are nearly the same so that there would be no difference using either Tantalo (1998) or Marigo et al. (1999) models. Now the formation of C-stars occurs for initial masses larger than $1.5 M_{\odot}$.

(d) Finally, for the sake of simplicity the possibility of a late O-rich phase is taken into account only for $Z=0.004$ and $Z=0.008$ and is ignored at higher metallicities owing to the very small difference between the maximum luminosity of the AGB phase and of the C-star regime. See for instance the case of $Z=0.02$ in Fig. 5 .

Basing on the above considerations the use of the Tantalo (1998) isochrones is fully adequate to our aim of studying the effect of the dusty envelope on processing the radiation coming from the central object. Therefore we will adopt the Tantalo (1998) models as source of our SSPs and use the models by 
Marigo et al. (1999) only to fix the luminosity at which the transition from O-rich to C-stars occurs. Work is in progress to replace the SSPs by Tantalo (1998) models with new ones fully incorporating the TP-AGB stars as modeled by Marigo et al. (1999).

\subsection{A remark on the libraries of stellar spectra and the evolutionary models of AGB stars}

The general accuracy of the final result depends on the adopted libraries of stellar spectra, in particular the correlation between the theoretical parameters $T_{\text {eff }}$, gravity and chemical abundance and the associated SED, and important details of the evolutionary models for AGB stars, in particular the dredgeup episodes altering the surface chemical abundances, the efficiency of mass-loss terminating the AGB evolution, the path of AGB stars in the HR-diagram eventually determining the $T_{\text {eff }}$ and luminosity of the AGB stars and the associated SEDs in turn, and finally the accuracy of the isochrone construction and of the stellar models underneath. To summarize and review in detail all the sources of the various items above would lead to too a lengthy discussion which goes perhaps beyond the scope of this study. Suffice it to mention, that the libraries of stellar spectra, isochrones, SSPs in usage here have been successfully tested against, somehow tailored to match, and applied to study the broad band $U B V R I$ photometric data of star clusters (stellar populations in general), e.g. Girardi \& Bertelli (1998), Girardi (1999), Carraro et al. (1999), Tantalo et al. (1998). As far as the evolutionary models of AGB stars are concerned, the same considerations hold good, because they have been calibrated on the observational data, e.g. the luminosity function of C-stars in the Magellanic Cloud (Marigo et al. 1999). To conclude the libraries of stellar spectra and evolutionary models of AGB stars we have adopted are fully adequate to our purposes. It is, however, long known that the IR colors of the classical models of AGB stars disagree with the observational data. In addition to improving the physics of the models, for instance by including the effect of C-rich composition on the opacity of the outermost layers, see Marigo (2002) which would yield AGB models of much cooler $T_{\text {eff }}$ during the C-rich phase (these models are not yet incorporated in this study), the dust shells are expected to strongly affect the IR colors and to bring them to better agree with the observations. This indeed is the aim of this study.

\section{Infrared spectra of SSPs}

In Fig. 6 we plot the SED of dusty SSPs for different values of the age limited to the case with metallicity $Z=0.008$ and in Fig. 7 we show the same but for the classical SSPs in which the effect of dust is neglected. The displayed SEDs are for ages in the range 0.25 to $10 \mathrm{Gyr}$. A better view of the difference brought by dust is shown in Fig. 8, in which the new and old SEDs are compared for a few selected ages. The differences are remarkable. First of all, in the old SSPs the spectra do not extend into the medium and far IR (MIR and FIR, respectively), but sharply decline for wavelengths longer than about 3-4 $\mu \mathrm{m}$. In contrast, the spectra of the new SSPs extend toward

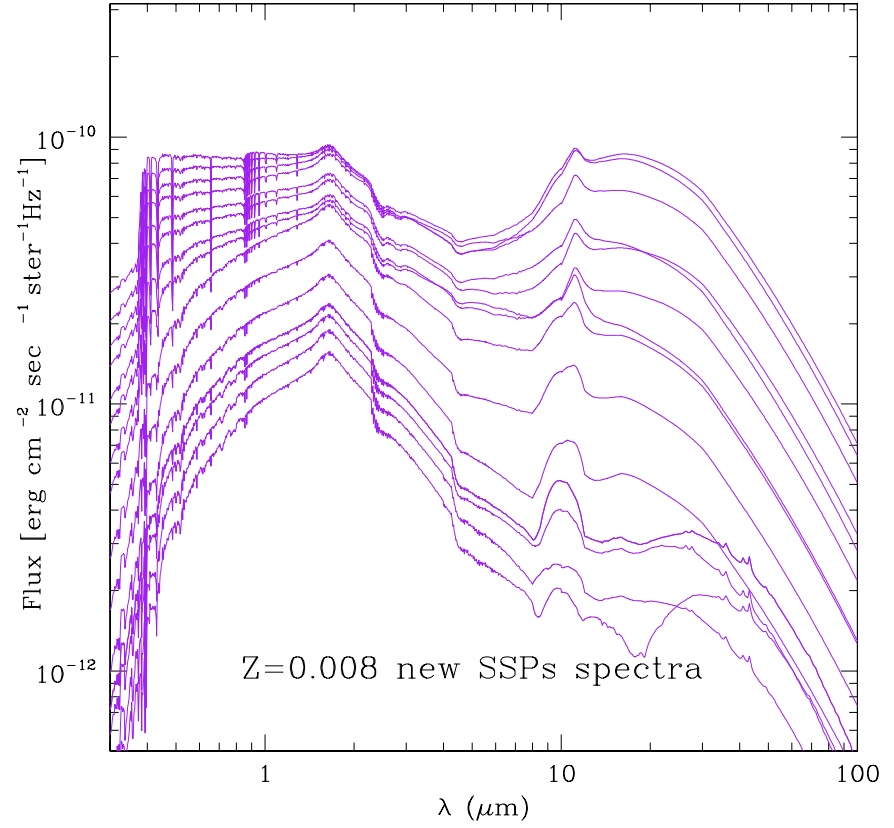

Fig. 6. Integrated SEDs $F_{v}$ vs. $\lambda$ for the SSPs with $Z=0.008$, ages from 0.25 to $10 \mathrm{Gyr}$ and the inclusion of dusty circumstellar envelopes in AGB stars. From the bottom to the top the displayed ages are: 10, $7.5,5,4,3,2,1.5,1,0.8,0.6,0.5,0.4,0.35,0.3$, and $0.25 \mathrm{Gyr}$.

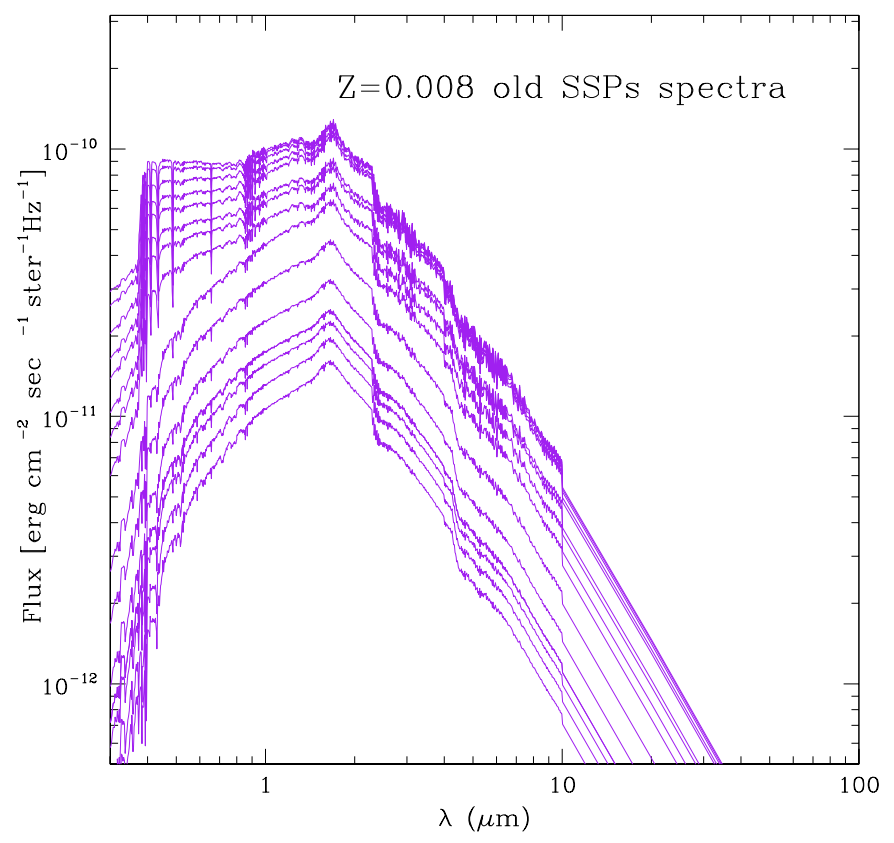

Fig. 7. The same as in Fig. 6 but for the classical SSPs of Tantalo (1998).

long wavelengths and the flux is considerable also in the MIR and FIR.

The differences start at about $1 \mu \mathrm{m}$ and in the IR range up to 3-4 $\mu \mathrm{m}$ the flux of dusty SSPs is lower than the old one: this is a consequence of the fact that dusty envelopes shift the emission of $\mathrm{M}$ and $\mathrm{C}$ stars toward longer wavelengths. The amount of energy shifted to longer wavelengths is larger for the young ages, because more massive and luminous AGB stars are present. 


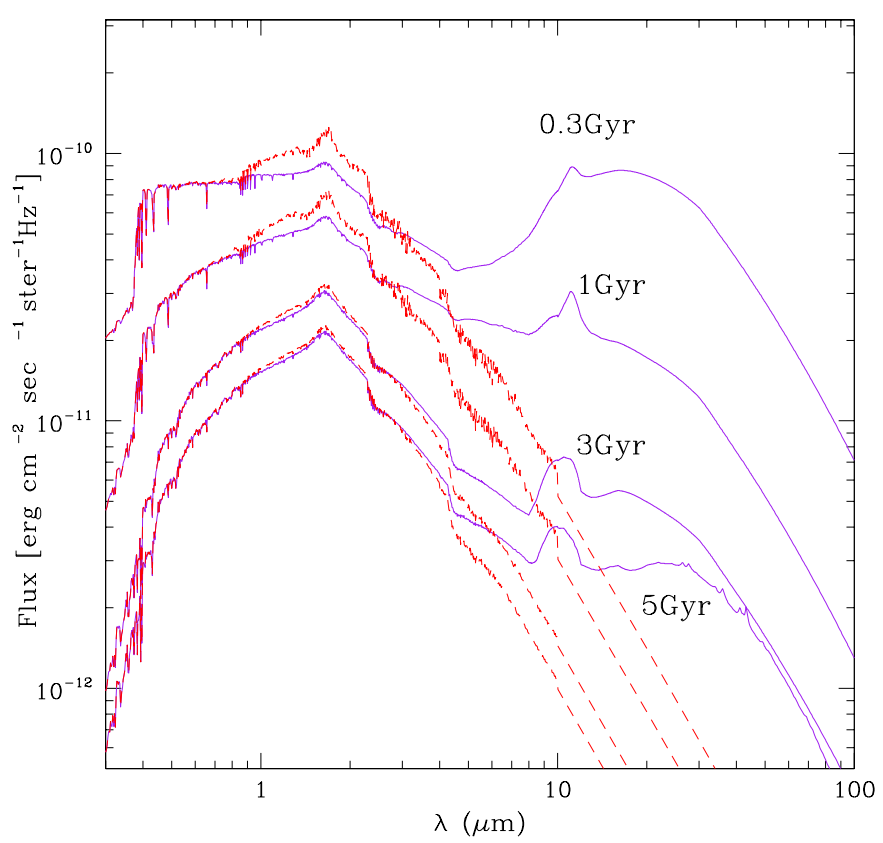

Fig. 8. Detailed comparison of SEDs $F_{\lambda}$ vs. $\lambda$ for the old (dashed lines) and new SSPs (continuous lines) with $Z=0.008$. Only four ages are considered as indicated.

It is worth noticing the different IR spectrum of the new SSPs, the evolution of the features at $11.3 \mu \mathrm{m}$ and $9.7 \mu \mathrm{m}$ in particular. For young ages, e.g. the SSPs of 0.3 Gyr and 1 Gyr, the spectrum does not exhibit features due to crystalline silicates, because the C-stars dominate (the $11.3 \mu \mathrm{m}$ feature of $\mathrm{SiC}$ is indeed prominent); for intermediate ages, such as $3 \mathrm{Gyr}$, the O-stars of low optical depth influence the spectrum and the $9.7 \mu \mathrm{m}$ feature can be seen in emission. For older ages, from 5 Gyr onward, the O-stars dominate, so the spectrum becomes more articulated and the features due to crystalline silicates start to appear at long wavelengths in the IR.

In Fig. 10 we plot the detailed evolution of the $\mathrm{SiC}$ and $\mathrm{Si}-\mathrm{O}$ stretching mode features at increasing age. At young ages $(0.3,0.5 \mathrm{Gyr})$ there is only the feature at $11.3 \mu \mathrm{m}$ of the $\mathrm{SiC}$; at about at $1 \mathrm{Gyr}$, the $9.7 \mu \mathrm{m}$ of $\mathrm{Si}-\mathrm{O}$ starts to appear; in the age range 1 to 2 Gyr the two features overlap; finally, at older ages the $11.3 \mu \mathrm{m}$ of $\mathrm{SiC}$ disappears, and only the feature at $9.7 \mu \mathrm{m}$ of $\mathrm{Si}-\mathrm{O}$ occurs.

In order to illustrate the effect of the metallicity, we plot in Fig. 9 the SEDs of the case with $Z=0.02$ for the same ages of the case with $Z=0.008$ shown in Fig. 6 . The evolution of the spectrum is similar but we can observe an interesting change for the youngest ages where the $9.7 \mu \mathrm{m}$ feature is slightly in absorption rather than in emission as at older ages. The explanation of it can be seen in Fig. 5. For the youngest ages, the luminosity interval in which C-stars appear becomes very thin, and the stars at the AGB tip, with the highest mass-loss rate and the highest optical depth, are O-stars. So the SSP spectrum becomes dominated by the $9.7 \mu \mathrm{m}$ feature in absorption and not in emission, as expected in envelopes with high optical depth. A similar situation would also occur at lower metallicities (e.g. $Z=0.008$ ), but only at ages younger than $0.2 \mathrm{Gyr}$ as shown by the luminosity intervals for C-stars displayed in Fig. 4.

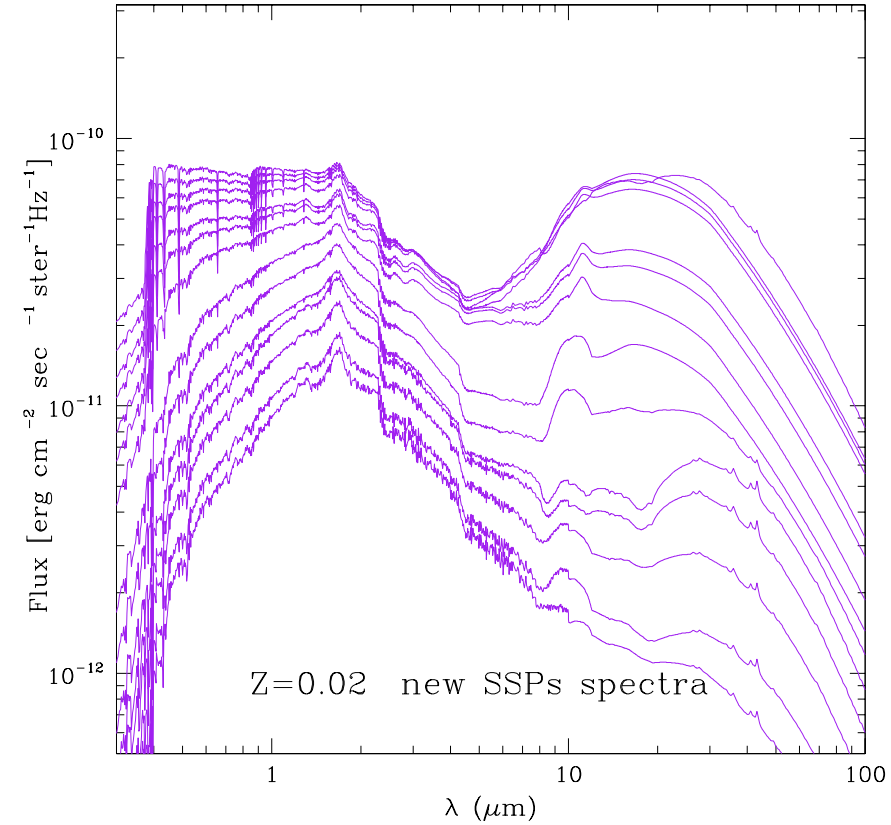

Fig. 9. The same as in Fig. 6 but for new SSPs with $Z=0.02$.

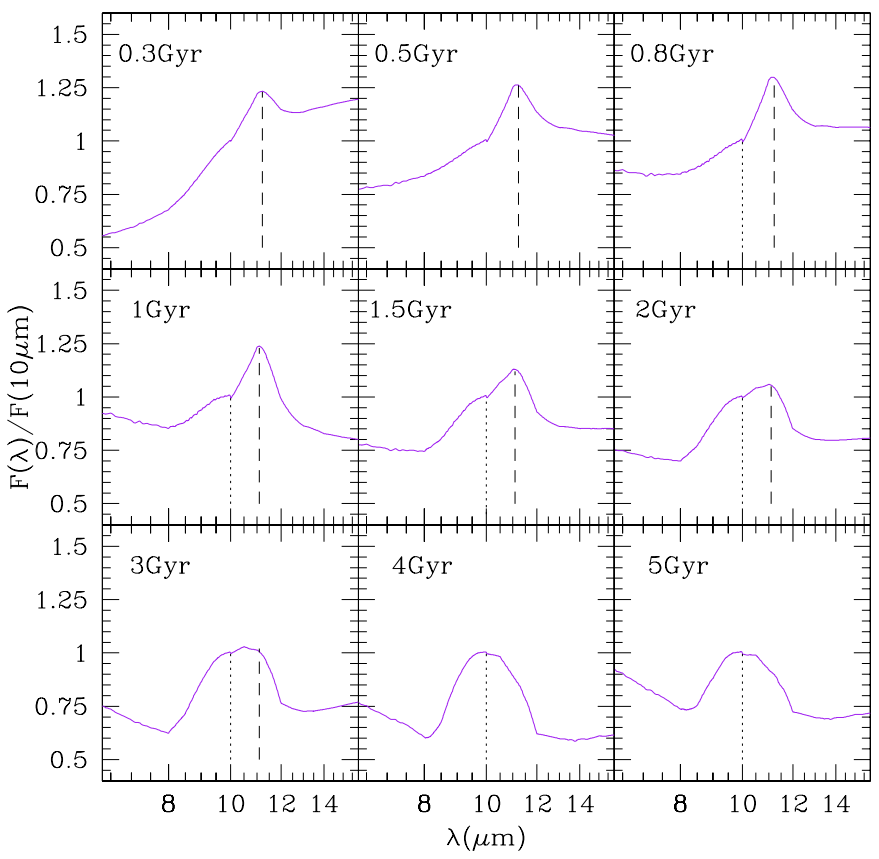

Fig. 10. Evolution of the $11.3 \mu \mathrm{m}$ feature of $\mathrm{SiC}$ (dashed vertical lines) and the $9.7 \mu \mathrm{m}$ feature of stretching vibrations of $\mathrm{Si}-\mathrm{O}$ bonds (dotted vertical lines) caused by the stretching modes of this molecule in SSPs of metallicity $Z=0.008$ and ages going from $0.3 \mathrm{Gyr}$ to $5 \mathrm{Gyr}$.

Silva et al. (1998) calculated spectra of SSPs with AGB dusty shells for chemical mixtures containing either silicate or graphite grains. They do not consider the more realistic situation in which both types of grain are present and do not include the transition of O-stars to C-stars (followed in some circumstances by a late transition from $\mathrm{C}$-stars back to $\mathrm{O}$ stars for AGB stars of very high mass). Thanks to the inclusion of the transition luminosities of Marigo et al. (1999), it is now possible to follow the IR-SED at changing the proportions of C-stars with respect to O-stars, which is expected to vary with the evolution of the AGB star population. 


\section{Broad-band colors in the infrared}

To test how the presence of dusty shells around AGB stars would affect the SEDs and colors of individual objects, we derive IRAS and Johnson broad-band colors and compare them with the observational data for a selected sample of AGB stars of different type. In addition to this we calculate the integrated colors of SSPs in the Johnson system and compare them with a sample of star clusters of the Magellanic Clouds.

IRAS colors. To derive the theoretical IRAS monochromatic fluxes we have convolved the SEDs of our SSPs with IRAS transmission curves, derived from the IRAS Explanatory Manual Supplement (on line at http: //space.gsfc.nasa.gov/astro/iras/docs/exp. sup/). Following Bedijn (1987), the monochromatic flux is defined as

$F_{\lambda}=\frac{\int F_{\lambda} \Phi_{\lambda} \mathrm{d} \lambda}{\int\left(\frac{F_{\lambda}^{\mathrm{a}}}{F_{0 \lambda}^{\mathrm{a}}}\right) \Phi_{\lambda} \mathrm{d} \lambda}$

where $\Phi_{\lambda}$ is the instrumental profile, $F_{\lambda}$ is the theoretical flux distribution, $F_{\lambda}^{\mathrm{a}}$ is the assumed flux distribution, that for IRAS bands is $\propto \lambda^{-1}$, and $F_{0 \lambda}^{\mathrm{a}}$ is the assumed flux distribution referred to the central wavelength of the band as defined in Neugebauer et al. (1984). If the source has a profile with a shape different from the dependence $\propto \lambda^{-1}$, one has to correct the flux using the so-called $K$ factor given by

$$
K=\frac{\int\left(\frac{F_{v}}{F_{v_{0}}}\right) \Phi_{\nu} \mathrm{d} v}{\int\left(\frac{F_{\nu}^{\mathrm{a}}}{F_{v_{0}}^{\mathrm{a}}}\right) \Phi_{\nu} \mathrm{d} \nu}
$$

and to divide the monochromatic flux resulting from Eq. (32) by this factor. Finally, the IRAS colors are given by

$$
\begin{aligned}
& {[12-25]=-2.5 \log \left(\frac{F(12)}{F(12)_{0}}\right)+2.5 \log \left(\frac{F(25)}{F(25)_{0}}\right)} \\
& {[25-60]=-2.5 \log \left(\frac{F(25)}{F(25)_{0}}\right)+2.5 \log \left(\frac{F(60)}{F(60)_{0}}\right)}
\end{aligned}
$$

where $F$ (12), $F(25)$, and $F(60)$ are the IRAS theoretical fluxes in Jansky (Jy), and the constants of calibration $F(12)_{0}=$ $28.3 \mathrm{Jy}, F(25)_{0}=6.73 \mathrm{Jy}$, and $F(60)_{0}=1.19 \mathrm{Jy}$ are taken from the IRAS PSC Explanatory Manual Supplement (1988) ${ }^{4}$.

Our template sample of O-rich AGB stars (Miras, SemiRegular, Long Period Variables, OH/IR etc, indicated by the filled circles) and C-stars (open circles) is shown in the IRAS two color plane [25-60] vs. [12-25] of Fig. 11. The location of the stars in this diagram is widely used to establish the nature of the sources. van der Veen \& Habing (1988) have defined a region named the $\mathrm{VH}$-window occupied by late-type stars. In addition to this, many other diagnostic plots are used to discriminate between $\mathrm{O}$ stars and $\mathrm{C}$ stars. The two-colors plots are constructed combining in various ways IRAS fluxes with other IR pass-bands, see for instance Epchtein et al. (1987). In

\footnotetext{
${ }^{4}$ We have also calculated the flux $F(100)$ adopting the calibration constant $F(100)_{0}=0.43 \mathrm{Jy}$. No use of this quantity is made here.
}

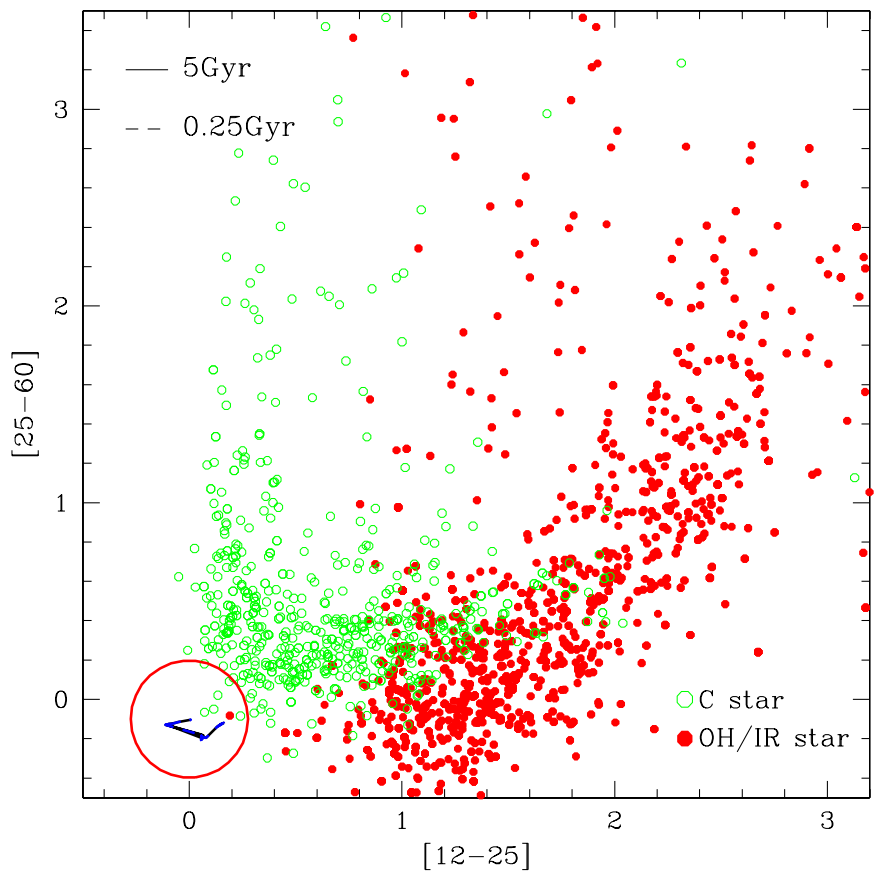

Fig. 11. The IRAS two color diagram [25-60] vs. [15-25]. The O-stars have been sampled from different sources: Lewis et al. (1990), Le Squeren et al. (1992), David et al. (1993), Blommaert et al. (1993), Chengalur et al. (1993), Loup et al. (1993), Xiong et al. (1994), Lepine et al. (1995), Lewis (1997), van Loon et al. (1998). The sample of C-stars is from Epchtein et al. (1990), Egan \& Leung (1991), Chan (1993), Guglielmo et al. (1993), Volk et al. (1992), Groenewegen (1995), Guglielmo et al. (1998), Guglielmo et al. (1997). The fluxes of the stars in the samples are obtained using the IRAS Point Source Catalogue. Two groups of coeval AGB stars of different mass represented by the SSPs with age of $0.25 \mathrm{Gyr}$ (dashed line) and $5 \mathrm{Gyr}$ (solid line) are compared to the data. The large circle marks the color range spanned by these AGB stars. Classical models of AGB stars are not able to fit the observational color distribution.

this diagram we also plot the colors of the AGB stars. In order to show the whole range of colors spanned by these stars we make use of the SSPs: more precisely the colors displayed by an SSP would correspond to AGB stars of the same age but different initial mass. By varying the age of the SSPs we may cover the whole range of colors of AGB stars of any initial mass and age.

In Fig. 11 models of classical AGB stars, i.e. with no dusty shells around, would span a small range of colors approximately centered at about around [12-25] $\simeq 0$ and [25-60] $\simeq 0$ (the encircled area visualizes the region covered by two SSPs with age of 0.25 and $5 \mathrm{Gyr}$ ). As already pointed out by Silva et al. (1998), this happens because the SED of AGB stars,

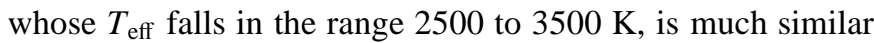
to the Rayleigh-Jeans distribution of black-body. The net result is that classical models of AGB stars fail to reproduce the observational distribution of $\mathrm{O}$ and $\mathrm{C}$-stars in the $\mathrm{VH}$-window.

Passing now to the new models of AGB stars with the dusty shell around, the situation is much improved. This is shown in Fig. 12, where now the colors of the AGB stars (represented by two SSPs of given age) stretch across the whole VH-window. As we expect, the path in the two color plot of 


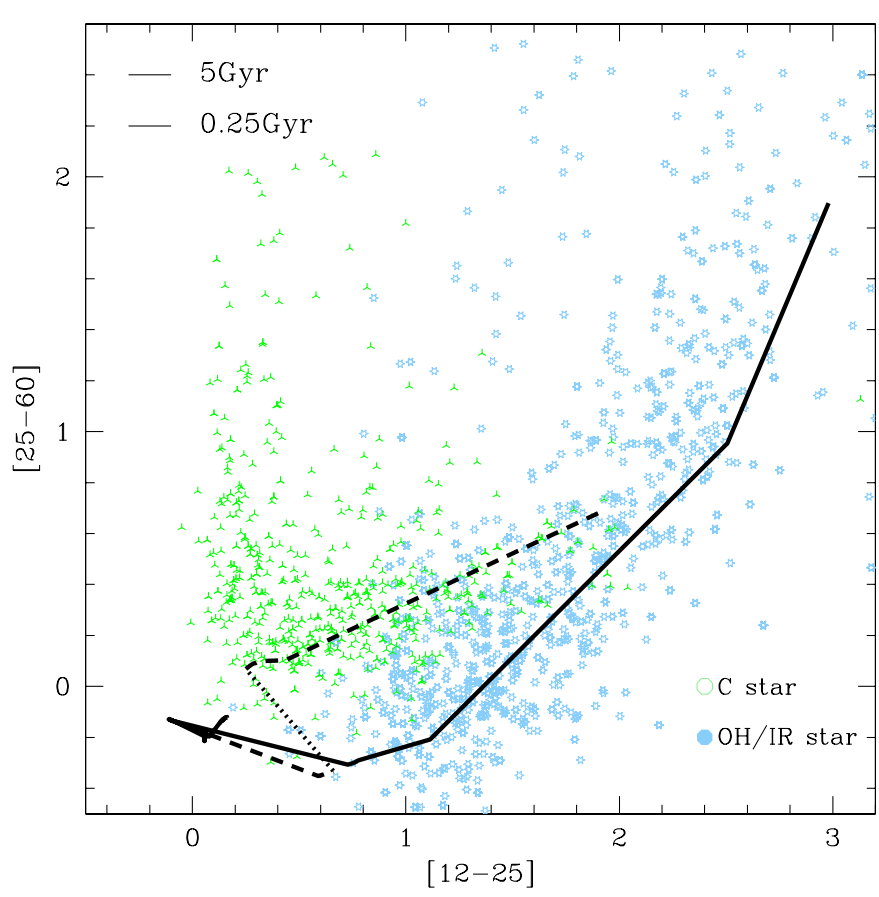

Fig. 12. The same as in Fig. 11 but in which two groups of new AGB stars of the same age and different initial mass are displayed. Like in Fig. 11 these AGB stars are simulated by two SSPs with age of $0.25 \mathrm{Gyr}$ (dashed line) and $5 \mathrm{Gyr}$ (solid line). Along the line for the young age we mark with a dotted line the rapid transition from $\mathrm{O}$ to C-stars.

massive AGB stars (the young SSP of 0.25 Gyr shown by the dashed line) and low-mass AGB stars (the old SSP of 5 Gyr shown by the solid line) is different. The low mass AGB stars overlap only the region occupied by O-stars, whereas the more massive AGB stars jump into the region crowed by C-stars when the transition from O- to $\mathrm{C}$-star occurs. This stage is marked by the dotted portion of the dashed line.

However, it must be noticed that many stars in our sample have [25-60] colors that are significantly redder than predicted by the theory.

This fact could be easily explained by the so-called cirrus contamination (Ivezic \& Elitzur 1995). In short the cirrus emission $C_{\lambda}$ may affect the IRAS fluxes because, owing to the point-like nature of the sources, sky-subtraction may not be accurate enough and some contamination by the cirrus light can be present and significant, so that some additional correction of the data is required The cirrus emission $C_{100}$ can be estimated from IRAS quantity cirr 3 , i.e. the surface brightness at $100 \mu \mathrm{m}$ around the point source: $C_{100}=1.2 \cdot \operatorname{cirr} 3 \mathrm{Jy}$. Ivezic \& Elitzur (1995), have shown that long-wavelength IRAS point source fluxes are unreliable when $\operatorname{cirr} 3 \gtrsim(1-5) \cdot F_{60}$. Basing on this, it is possible to clean the sample by removing all the sources with $\frac{\text { cirr } 3}{F_{60}} \gtrsim a$, where $1 \leq a \leq 5$. In Fig. 13 are shown the same data of Fig. 12 but in which all stars with $\frac{\text { cirr } 3}{F_{60}} \gtrsim 3$ have been removed. Now the theoretical colors much better reproduce the observed ones. In general at increasing the cirrus correction, the agreement between theory and data improves, but too few stars are left over and the comparison loses statistical significance.

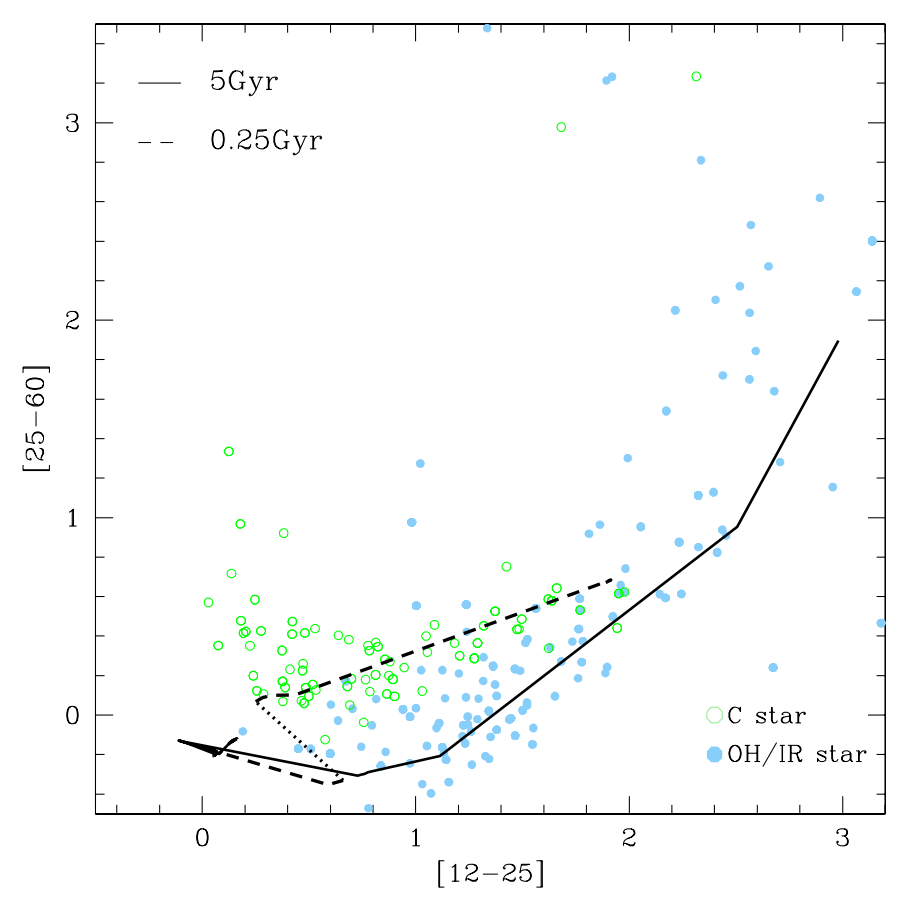

Fig. 13. The same as in Figs. 11 and 12 but in which the data have been selected imposing the condition $\frac{c i r r 3}{F_{60}} \leq 3$. All the stars with ratio $\frac{c i r r 3}{F_{60}}$ greater than 3 have been discarded because too much contaminated by the cirrus light.

Another point to note is that our models of AGB stars of different mass do not actually cover the whole color ranges of the data even considering the cirrus correction above. It is likely that the parameters adopted in the radiative transfer problem are not fully adequate. There are several causes worth being examined in some detail:

(i) Part of the disagreement can be due to the optical depth of the envelope and to its dependence on the chemical composition. Recalling the definition of optical depth, this could vary from model to model because of different compositions of the dusty envelopes. In this study we adopt different compositions of the dust for different intervals of optical depth, however we have not considered the possibility of different mixtures of silicate and carbon grains in envelopes with the same optical depth. This is a point of weakness because Ivezic \& Elitzur (1995) have demonstrated that mixed compositions could explain the spread of the data (see for example their Fig. 6).

(ii) Another possibility, is that the density profile of the matter is more complicated than a continuous power-law, as a consequence of discontinuous episodes of mass-loss of different intensity. Therefore the approximation $\rho \propto r^{-2}$ could be too simple, actually only mirroring average properties. Suh (1997) and Silva et al. (1998) have indeed shown that a narrow region of enhanced density, caused by a super-wind phase (Steffen \& Schönberner 2000) travelling across the envelope, could be the cause of a large spread in the two color diagram. We plan to examine this problem in a forthcoming study.

NIR colors. We also compare our theoretical colors in near IR, such as $[J-H],[H-K]$ and $[K-L]$ with the data in the same pass-bands for a sample of $\mathrm{O}$ - and C-stars. The comparison is shown in Figs. 14 and 15. Three groups of AGB stars 


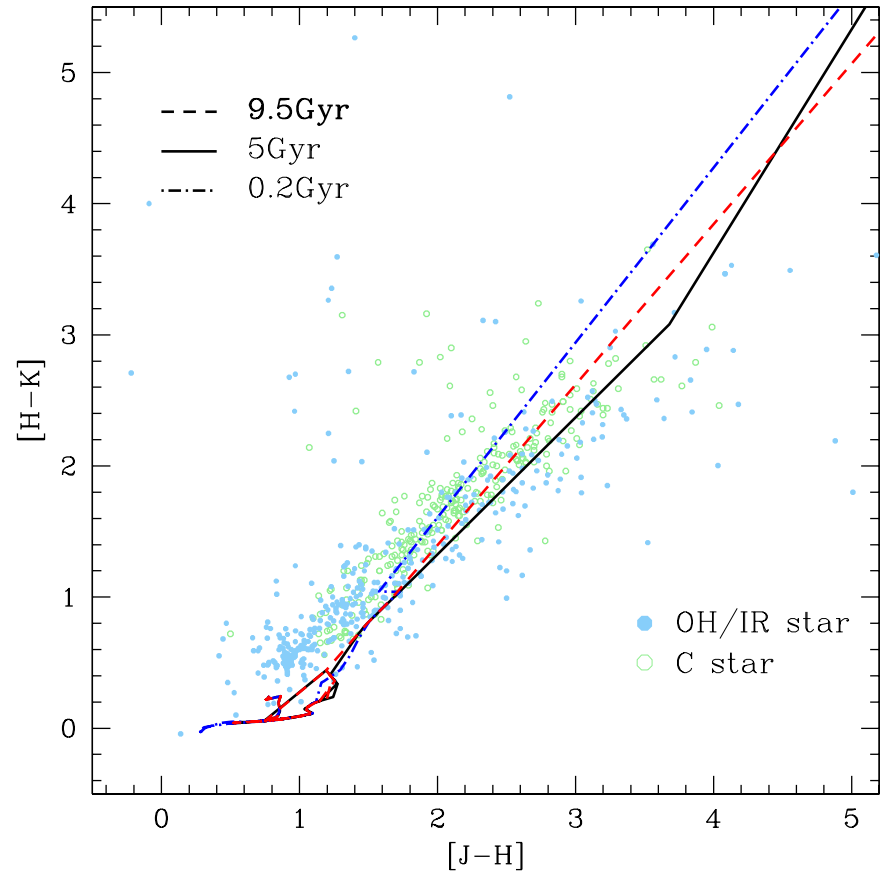

Fig. 14. The two color diagram $[H-K]$ vs. $[J-H]$ derived from the new models of AGB stars. The colors of the OH/IR and Mira stars are taken from Lepine et al. (1995), Xiong et al. (1994), Olivier et al. (2001), Whitelock \& Feast (1994) whereas those of the C-stars are derived from Epchtein et al. (1990), Guglielmo et al. (1993, 1997, 1998), Olivier et al. (2001). All the data are properly corrected for extinction. In particular, for the database of Lepine et al. (1995), the infrared data have been corrected with a new model of extinction (J. R. D. Lepine, private communication). The dotted-dashed, solid and dashed lines show three groups of AGB stars with age of $0.25,5$ and $9 \mathrm{Gyr}$ respectively.

are shown: massive objects (the SSP of $0.2 \mathrm{Gyr}$, dotted dashed line), intermediate mass AGB stars (the SSP of $5 \mathrm{Gyr}$, solid line), and low-mass AGB stars (the 9.5 Gyr SSP, dashed line). While the classical models for AGB stars are confined in a small region and thus fail to match the data, the new models with the dusty shells stretch across the whole region crowded by the observational data. Both in the $[J-H],[H-K]$ and in the $[J-K],[K-L]$ two color diagrams the fit is good, even if we still have some problem to fit the data of $\mathrm{O}$ stars. As noticed by Silva et al. (1998), problems in the fit could be due to the atmospheric models adopted for the M type spectra.

Star clusters. It might be worth of interest to compare the integrated broad-band colors of the SSPs whose AGB stars are enshrouded in the dust shell to those of a sample of star clusters. To this aim we have looked at the young globular clusters of the Large and Small Magellanic Clouds (LMC and SMC, respectively). Pietrzynski \& Udalski (2000) have presented age determinations for about 600 star clusters belonging to the central part of the LMC. These clusters are younger than $1.2 \mathrm{Gyr}$ and therefore the majority of them belong to the age range in which the AGB phase can develop significantly contributing to the integrated light of the cluster. Out of the catalog, we have selected a small sample in which the contribution to the total light by AGB stars is particularly strong. The

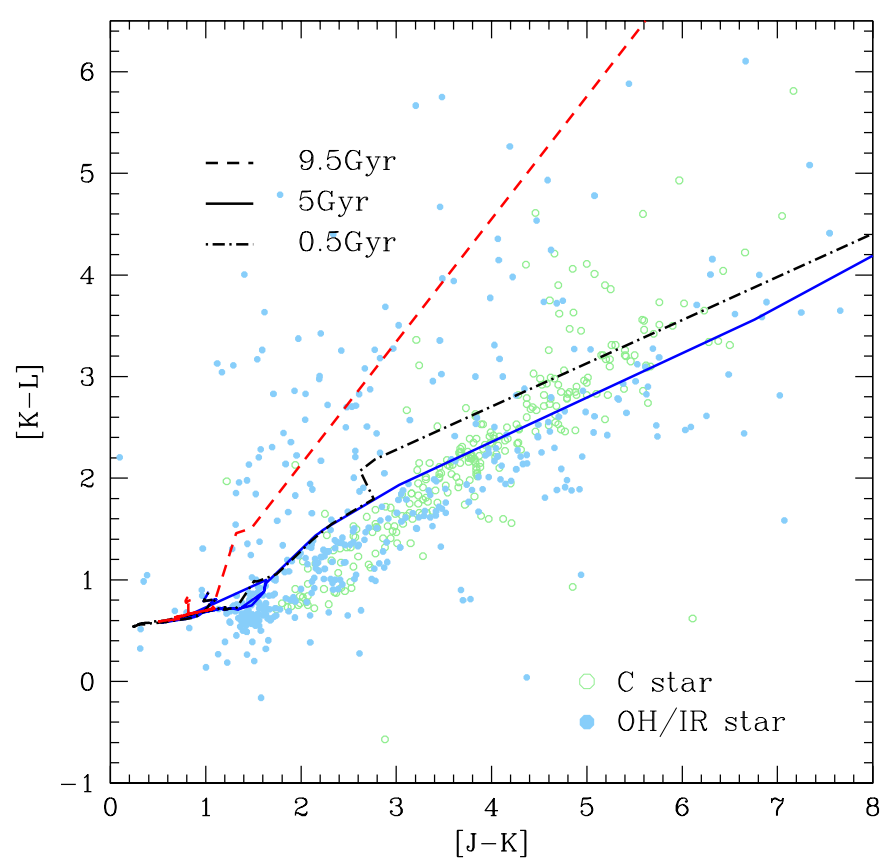

Fig. 15. The two color diagram $[K-L]$ vs. $[J-K]$. The sources of data and AGB models are the same as in Fig. 14.

photometric data of these clusters, are derived from the $2 M A S S$ Second Incremental Data Release and the companion Image Atlas, that contains about 1.9 millions of images in $J, H$ and $K_{\mathrm{S}}$ bands. The integrated magnitudes $J_{i}, H_{i}$ and $K_{i}$ have been calculated by Pretto (2002) and kindly made available to us. Finally, in order to compare our SSPs with those by Mouhcine \& Lançon (2002), we consider also the integrated IR colors for LMC and SMC clusters by Persson et al. (1983) used by Mouhcine \& Lançon (2002).

The series of Figs. 16-18 show the planes $[J-H]$ vs. $[H-K],[V-K]$ vs. $[H-K]$, and $[V-K]$ vs. $[J-K]$, respectively. In each diagram we display the data of Persson et al. (1983) for LMC (open circles) and SMC (filled circles) clusters, the LMC clusters calculated by Pretto (2002) (open squares), the SSPs by Mouhcine \& Lançon (2002), the old SSPs by Tantalo (1998), and the new ones of this study. The SSPs of Mouhcine \& Lançon (2002) and Tantalo (1998) on display span an age range from 100-150 Myr (the first ages at which the AGB contribution is significant) to $15 \mathrm{Gyr}$ and are for the metallicities $Z=0.008$, and $Z=0.02$. The new SSPs span the same range of ages and metallicities.

Looking at Figs. 16-18 we note that both classical and new SSPs generally agree with the data, even if in the $[J-H]$ vs. $[H-K]$ and $[V-K]$ vs. $[H-K]$ diagrams old SSPs span a narrower range in $[H-K]$ and are too red in $[J-H]$. In contrast the new SSPs have bluer $[J-H]$ and $[V-K]$ colors. Although the agreement is better than with the old SSPs, it is not yet satisfactory. An additional shift to bluer $[J-H]$ and $[H-K]$ is required. This would imply that the slope of the SSP SEDs ought to become steeper passing from the $J$ to the $K$ pass-band than allowed by the present models. Finally the SSPs by Mouhcine \& Lançon (2002) 


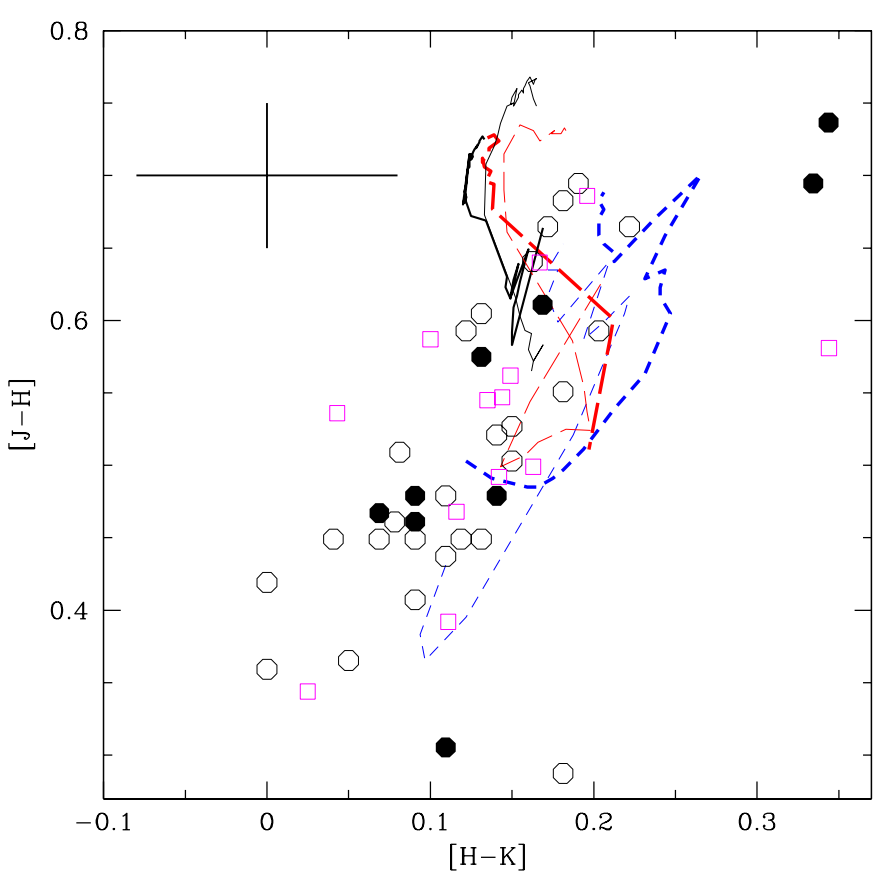

Fig. 16. The two color diagram $[J-H]$ vs. $[H-K]$ for young star clusters of the Magellanic Clouds. The open circles are the LMC clusters selected by Mouhcine \& Lançon (2002) from the catalog of Persson et al. (1983), whereas the filled circles are the same but for SMC clusters. The open squares are a few LMC clusters whose IR colors have been derived by Pretto (2002) using the data of the 2MASS Second Incremental Data Release and companion Image Atlas (see the text for more details). All the data have been properly reddening corrected. The lines show the color range spanned by SSPs of different metallicity and physical input: the thin and thick dashed lines are the SSPs of Mouhcine \& Lançon (2002) respectively for $Z=0.02$ and $Z=0.008$; the thin and thick long-dashed lines are our SSPs for $Z=0.02$ and $Z=0.008$; finally the thin and thick solid lines in the upper part of the diagram are the old SSPs by Tantalo (1998) for $Z=0.02$ and $Z=0.008$. The age of all SSPs is from $100-150 \mathrm{Myr}$ to $15 \mathrm{Gyr}$.

extend toward bluer colors than the other SSPs. A possible explanation of this unsatisfactory fit is that the theoretical models of $\mathrm{M}$ giants are not yet able to reproduce the empirical spectra of O and C stars used by Lançon \& Mouhcine (2002). It is worth recalling that the ultimate reason of the good agreement achieved by Lançon \& Mouhcine (2002) models is the adoption of empirical spectra. More work is required to improve the theoretical spectra of $\mathrm{O}$ and $\mathrm{C}$-stars to be included in population synthesis studies.

Temporal evolution. Finally we look at the temporal evolution of our theoretical colors. This is shown in Figs. 19 and 20 for the colors $[J-H],[J-K]$ and $[V-K]$. Figure 19 is for the metallicity $Z=0.008$, whereas Fig. 20 is for $Z=0.020$. The age range goes from the time at which the first AGB stars are formed up to very old ages when the contribution of AGB stars to the integrated flux gets very low. For the sake of comparison we show also the colors of the classical SSPs by Tantalo (1998) and Girardi et al. (2002) - these latter are based on more recent stellar models calculations and stellar libraries - and

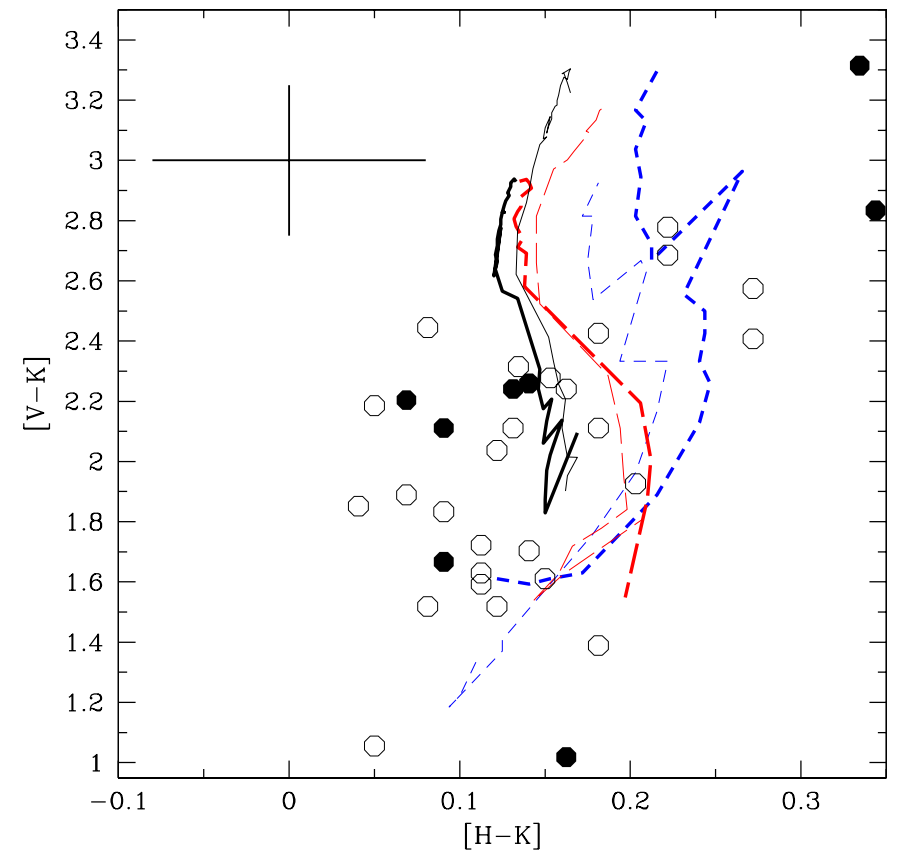

Fig. 17. The two color diagram $[V-K]$ vs. $[H-K]$ in the near IR. The meaning of the symbols is the same as in Fig. 16.

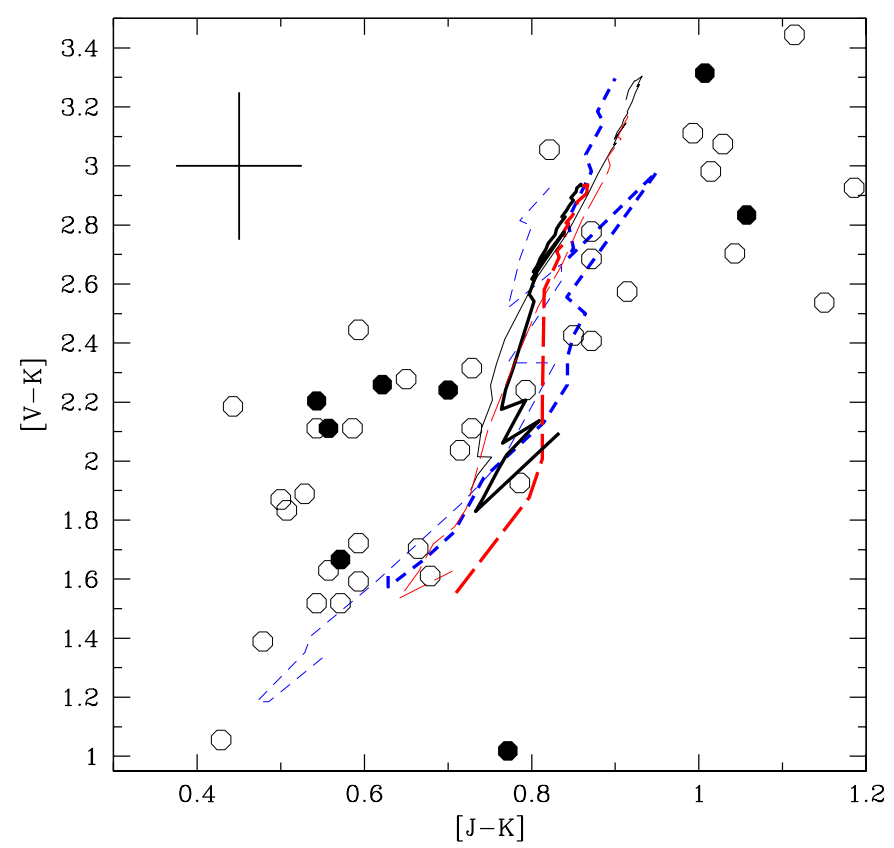

Fig. 18. The two color diagram $[V-K]$ vs. $[J-K]$ in the near IR. The meaning of the symbols is the same as in Fig. 16.

finally those of the SSPs by Mouhcine \& Lançon (2002). The following remarks can be made:

(i) The IR colors of the old SSPs by Tantalo (1998) differ from those of Girardi et al. (2002) and also from those of the present study.

(ii) The IR colors of the new SSPs marginally agree with the semiempirical ones by Mouhcine \& Lançon (2002). In particular we note that the IR colors are in good mutual agreement for ages older than about $1 \mathrm{Gyr}$, whereas for younger 


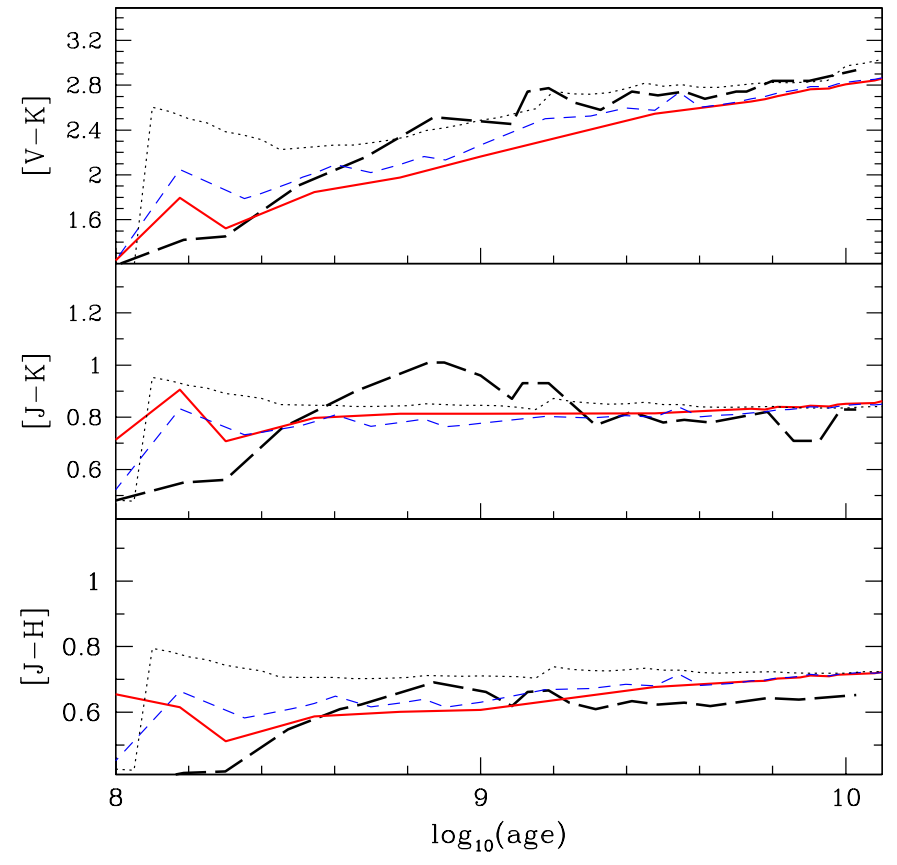

Fig. 19. The integrated colors $[J-H],[J-K]$, and and $[V-K]$ as function of the age in the range 0.1 to $20 \mathrm{Gyr}$ for SSPs with $Z=0.008$. The dashed lines are the old SSPs by Tantalo (1998). The thick solid lines are the SSPs of this study for the same metallicity. The dotted lines are the corresponding SSPs by Girardi et al. (2002). Finally, the thick long dashed line shows the SSPs of Mouhcine \& Lançon (2002) for $Z=0.008$.

ages, where the contribution of AGB stars is important, they can significantly differ.

(iii) Finally, it is worth noticing that IR colors from different sources offer an embarrassing picture. Those of Girardi et al. (2002) are redder than any other, except for the $[J-K]$ bump in Mouhcine \& Lançon (2002) at about 1 Gyr. The colors of Tantalo (1998) are bluer than those of Girardi et al. (2002) and corrected for dust effect surprisingly they get even bluer, with some exceptions. So, the colors of Girardi et al. (2002), Tantalo (1998) and ours form a sequence from red to blue colors that deserves a careful analysis. First of all we have to clarify the difference between Tantalo (1998) and Girardi et al. (2002) without shells of dust.

Spectral libraries. A plausible cause of disagreement between Girardi et al. (2002) IR colors and Tantalo (1998) could be the different libraries of stellar spectra in usage. Girardi et al. (2002) have adopted a library in which some empirical spectra for $\mathrm{M}$ giant stars are included (see Girardi et al. 2002, for all details). Tantalo (1998) and this study adopt the Lejeune et al. (1998) library in which purely theoretical spectra (even for M-type stars) are included. To check the effect of different spectral libraries we have taken the isochrones/SSPs of Girardi et al. (2002) and recalculated the IR colors using both the Girardi et al. (2002) and Lejeune et al. (1998) libraries. The results are compared in the bottom panel of Fig. 21 limited to the case of the $[J-H]$ color. Assigned the set of isochrones/SSPs, no significant difference arises passing from the library of Girardi et al. (2002) to that of Lejeune et al. (1998).

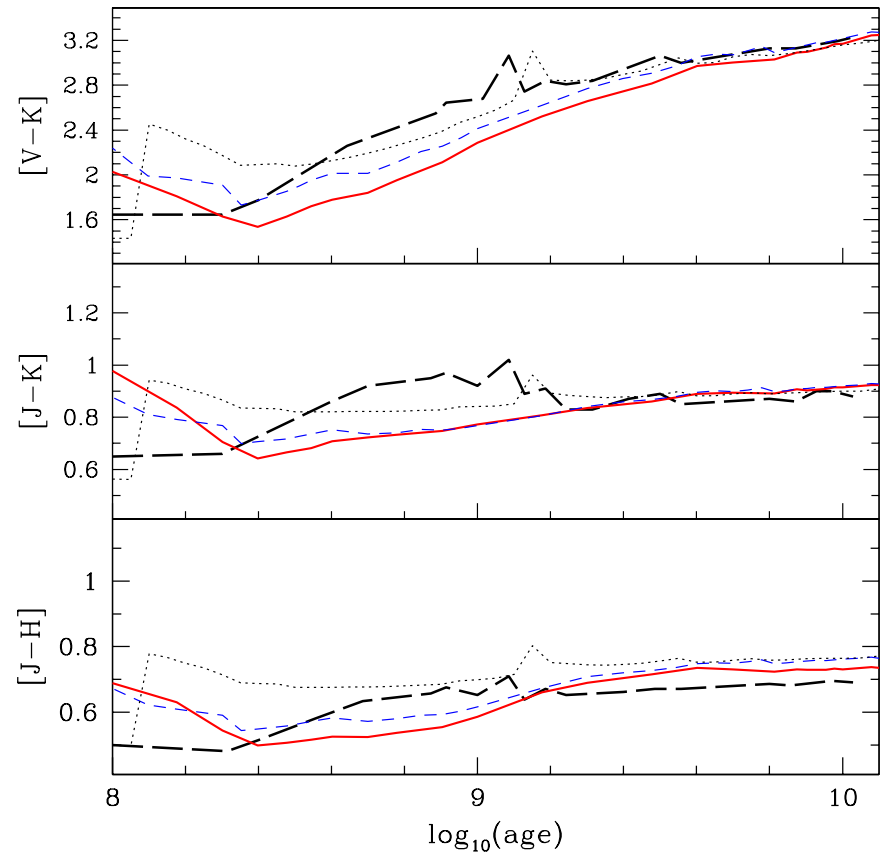

Fig. 20. The integrated color $[J-H],[J-K]$, and and $[V-K]$ as function of the age in the range 0.1 to $20 \mathrm{Gyr}$ for SSPs with $Z=0.02$. The meaning of the symbols is the same of Fig. 19.

Models of AGB stars. Therefore, the cause of disagreement in the IR colors of Figs. 19 and 20 could be in the different models for the AGB phase:

(a) Mass-loss. The first important parameter to look at is the rate of mass-loss. The SSPs of Girardi et al. (2002), Tantalo (1998) and of our study stand on the Vassiliadis \& Wood (1993) prescription (super-wind included), but for the delay correction proposed by Vassiliadis \& Wood (1993) which is not included in Girardi et al. (2002). The effect of mass-loss has therefore little influence on the colors difference.

(b) Path in the HRD. Another source of disagreement could be the distribution of AGB stars in the HR-diagram. To this aim we compare in top panel of Fig. 21 the locus predicted by three sets of isochrones/SSPs, i.e. Bertelli et al. (1994), Tantalo (1998) and Girardi et al. (2002). The comparison is limited to the age of $0.3 \mathrm{Gyr}$ and solar metallicity $(Z \simeq 0.02)$. At this age the contribution of the AGB to IR colors is important and the difference among the sources of IR colors is stronger. We note that while the Girardi et al. (2002) and Bertelli et al. (1994) AGB is a straight line (the one of Girardi et al. 2002, is even bluer and fainter than Bertelli et al. 1994), the AGB of Tantalo (1998) first coincides with Bertelli et al. (1994) and eventually bends to cooler $T_{\text {eff }} \mathrm{s}$. All this can be explained by the slightly different physics adopted in the underlying stellar models. Girardi et al. (2002) adopt the same rate of mass-loss as in Tantalo (1998) but a higher mixing length parameter compared to Bertelli et al. (1994) and Tantalo (1998). The higher mixing length given by Girardi et al. (2002) is the result of the lower, more recent opacities, and the calibration of their stellar models on the Sun (see Girardi et al. 2002, for all details). Bertelli et al. (1994) and Tantalo (1998) used the same stellar models and mixing length in turn, but adopted different expressions for 

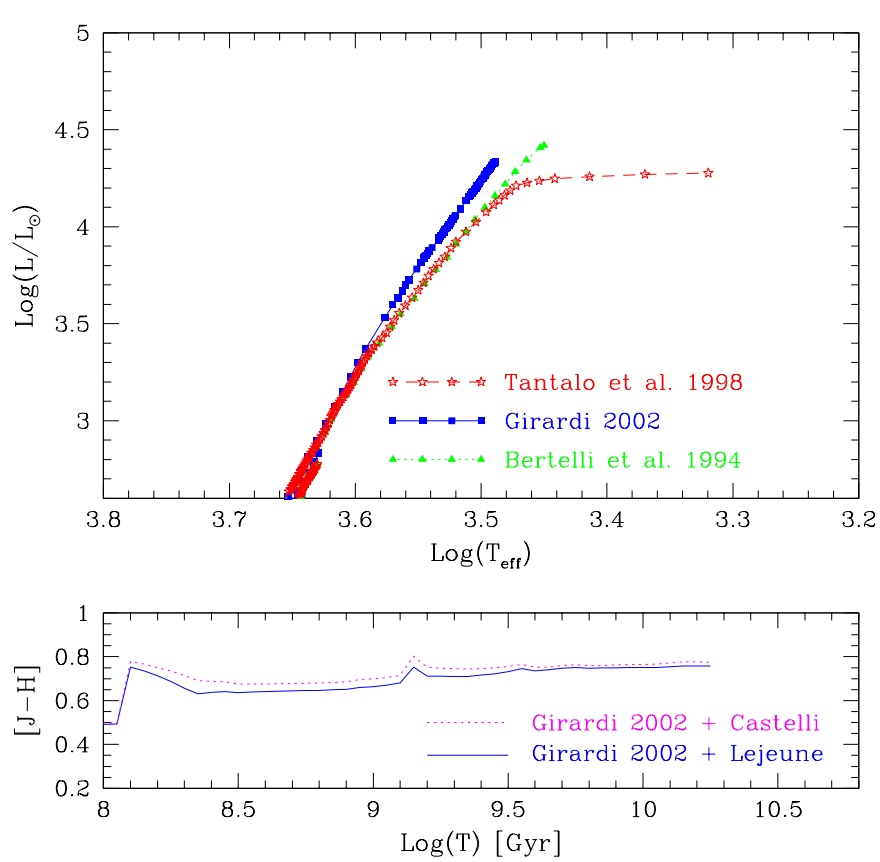

Fig. 21. Upper panel: comparison between the AGB of various isochrones set. We plot the AGB for old Bertelli et al. (1994) isochrones (filled triangles), Tantalo (1998) isochrones used in this work (open stars), new Girardi et al. (2002) isochrones (filled squares). Lower panel: comparison between $[J-H]$ colors of Girardi et al. (2002) SSPs calculated with Girardi et al. (2002) library of stellar spectra and with Lejeune et al. (1998) theoretical library.

the mass-loss rate and the relationship connecting luminosity, $T_{\text {eff }}$ and core mass ( $\left.\mathrm{L}-T_{\text {eff }}-\mathrm{Mc}\right)$. The rate of mass-loss adopted by Bertelli et al. (1994) is indeed lower than the one used by Tantalo (1998). This explains why their AGB phase extends to bright luminosities. The ( $\mathrm{L}-T_{\text {eff }}-\mathrm{Mc}$ ) relationship adopted by Bertelli et al. (1994) stand on the previous studies by Bertelli et al. (1990) and Groenewegen \& de Jong (1993), whereas that used by Tantalo (1998) incorporates the TP-AGB models by Marigo et al. (1996). This accounts for the different slope in the HR-diagram of the late AGB phase passing from Bertelli et al. (1994) to Tantalo (1998). Incidentally the (L- $\left.T_{\text {eff }}-M c\right)$ relationship adopted by Girardi et al. (2002) is much similar to that of Bertelli et al. (1994). The different path in the HR-diagram bears very much on the final IR colors, because cooler AGB stars should imply more flux at longer IR wavelengths, so that a bluer $[J-H]$ color is expected: indeed we have that very cool AGB stars by Tantalo (1998) yield bluer $[J-H]$ colors, whereas the hotter AGB stars by Girardi et al. (2002) yield redder colors.

(c) Number of $A G B$ stars. Finally, the relative number of AGB in a SSP must surely play a role. To probe this effect, in Fig. 22 we plot for the SSP with solar metallicity and age of 0.3 Gyr the cumulative contribution to the total flux by stars in different evolutionary stages. Five steps are considered: up to turn-off (TO), from the turn-off up to the tip of the RGB (T-RGB), from this up to the end of the He-burning phase (HeB), and from this up the end of the AGB (AGB), and finally the last phase leading to the formation of Planetary Nebulae and White Dwarf (this latter is not included in our

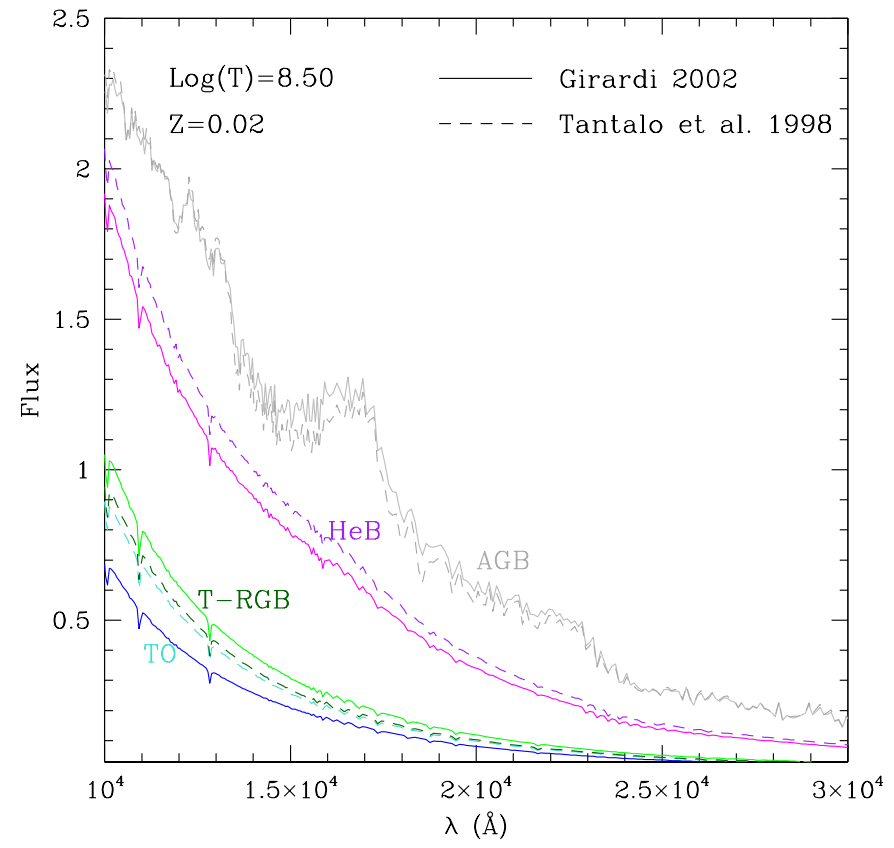

Fig. 22. The flux emitted by a SSP with solar metallicity $Z=0.02$ and age of 0.3 Gyr from the stars in different evolutionary stages. We split the contribution to the total flux into five phases: main sequence (TO), from TO until RGB tip (T-RGB), horizontal branch (HB), asymptotic giant branch (AGB) and finally planetary nebulae (PN) that are not represented for the sake of clarity. The dashed lines are for the Tantalo (1998) SSP, whereas the solid lines are same but for the SSP of Girardi et al. (2002).

analysis owing to the very short lifetime). Lumping together all stages up to $\mathrm{HeB}$ we note that the cumulative flux predicted by Girardi et al. (2002) is lower than that predicted by Tantalo (1998). The situation is reversed at the final step when the contribution from AGB inclusion stars is added: Girardi et al. (2002) SSPs have higher fluxes than Tantalo (1998). This means that a higher percentage of AGB stars is predicted by Girardi et al. (2002). The result is confirmed by looking at the tabulations of the integral of the IMF along the isochrones, the so called FLUM defined by Bertelli et al. (1994) to whom the reader should refer for details. The reason of it is the kind of (L- $\left.T_{\text {eff }}-\mathrm{Mc}\right)$ relationship adopted by Girardi et al. (2002) which ultimately drives the evolutionary rate (relative number of stars) during the AGB phase. So, we can conclude that the combined effect of the path in the HRD and the relative number of AGB explain the redder colors of Girardi et al. (2002).

Effects of dust. Finally, there is a fundamental question to be clarified. Why the inclusion of AGB dusty shells on the Tantalo (1998) isochrones/SSPs makes their IR colors bluer than those of Girardi et al. (2002) instead of making them redder? The explanation can be found in the effect of the shells of dust on the stellar radiation of AGB stars. Dust shifts the flux from $J, H$ and $K$ bands to longer wavelengths, but in general its effect is stronger at the shorter wavelengths ( $J$ band) than at the longer ones ( $K$ band). More flux is driven away from the shorter wavelengths than the longer ones and this shift tends to increase the $J$ magnitude more than the $H$ and $K$ magnitudes. 
The energy shift changes the flux ratio in a complicated way thus making it slightly higher or smaller than before. If the flux ratio is higher the presence of the dusty shells yields the effect of a redder color: for example we can observe as the $[J-H]$ color tend to be bluer than one would expect from simple-minded considerations. For $[V-K]$ color the effect is simpler because dust increases only the $K$ magnitude without changing the $V$ magnitude and thus producing bluer colors.

\section{Summary, discussion and conclusions}

This paper provides accurate models for the dusty shells surrounding AGB stars and the re-processing of the radiation emitted by the central object into the far IR. The aim is to derive realistic SEDs of AGB stars and SEDs of SSPs to be used in studies of the colors of individual late-type stars and the integrated colors of stellar aggregates going from star clusters to galaxies.

The models for the dusty shells take into account the physical (density and temperature profiles and optical depth) and chemical structure (dust grains of different compositions as appropriate to the evolutionary phase under consideration) and solve the radiative transfer equations to determine the shift of part of the coming radiation into the far IR region of the spectrum. Particular attention is payed to the transition of an AGB star from the O-rich to the $\mathrm{C}$-rich regime taking into account recent theoretical models of AGB stars.

The SEDs of AGB stars are then convolved with the IRAS and Johnson-Cousins broad-band pass-bands to derive the IR and far IR colors to be compared with the observational data for individual $\mathrm{O}$ - and $\mathrm{C}$-stars. The same is made for the SEDs of SSPs to be compared to the integrated colors of star clusters. Agreement between theory and data is good if the effect of the dusty shells is considered.

Finally, we compare the age dependence of the integrated colors in the near IR for different sources of SSPs based on different physical input, i.e. Tantalo (1998), Girardi et al. (2002), Mouhcine \& Lançon (2002) and ours. While the various sources more or less agree for the oldest ages, they differ for the youngest ages, when there is significative contribution from AGB stars. The discrepancy can be ascribed to subtle details of the models for AGB stars proposed by different authors and to the use of empirical or theoretical libraries for AGB stars. To conclude, more work is required to derive magnitudes and colors of both single stars and SSPs in the near and far IR.

Extensive tabulations of spectral energies distributions and integrated magnitudes and colors for SSPs are available from the authors upon request.

Acknowledgements. We would like to thank P. Marigo for very useful discussions and for providing us the transition luminosities from O-rich to C-rich AGB stars. We are also grateful to C. Jaeger for making available to us the absorption coefficients of the forsterite and to J. R. D. Lepine for correcting data with his new model for the extinction. Finally, we would like to thank the anonymous referee for his/her constructive remarks. This study has been financed by Italian Ministry of Education and Research (MIUR) and the Padua University with the special contract "Formation and evolution of elliptical galaxies: the age problem".

\section{References}

Allard, F., \& Hauschildt, P. H. 1995, ApJ, 445, 433

Bagnulo, S., Doyle, J. G., \& Griffin, I. P. 1995, Ap\&SS, 224, 425

Bedijn, P. J. 1987, A\&A, 186, 136

Begemann, B., Dorschner, J., Henning, T., et al. 1997, ApJ, 476, 199

Bernatowicz, T. J. 1997, in From Stardust to Planetesimals, ASP Conf. Ser., 122, 227

Bertelli, G., Bressan, A., Chiosi, C., Fagotto, F., \& Nasi, E. 1994, A\&AS, 106, 275

Bertelli, G. R., Betto, A. B., Chiosi, C., Nasi, E., \& Vallenari, A. 1990, A\&AS, 85, 845

Bessell, M. S., Brett, J. M., \& Scholz, M. W. P. R. 1991, A\&AS, 89, 335

Bessell, M. S., Brett, J. M., Wood, P. R., \& Scholz, M. 1989, A\&AS, 77,1

Blanco, A., Borghesi, A., Fonti, S., \& Orofino, V. 1998, A\&A, 330, 505

Blommaert, J. A. D. L., van der Veen, W. E. C. J., \& Habing, H. J. 1993, A\&A, 267, 39

Bohren, C. F., \& Huffman, D. R. 1983, Absorption and Scattering of Light by Small Particles (Wiley: New York), 530

Bressan, A., Chiosi, C., \& Fagotto, F. 1994, ApJS, 94, 63

Bressan, A., Granato, G. L., \& Silva, L. 1998, A\&A, 332, 135

Carraro, G., Girardi, L., \& Chiosi, C. 1999, MNRAS, 309, 430

Chan, S. J. 1993, PASP, 105, 1107

Chengalur, J. N., Lewis, B. M., Eder, J., \& Terzian, Y. 1993, ApJS, 89, 189

David, P., Lesqueren, A. M., \& Sivagnanam, P. 1993, A\&A, 277, 453

David, P., \& Papoular, R. 1990, A\&A, 237, 425

Dorschner, J., Friedemann, C., \& Guertler, J. 1978, Astron. Nach. 299, 269

Draine, B. T., \& Lee, H. M. 1984, ApJ, 285, 89

Egan, M. P., \& Leung, C. M. 1991, ApJ, 383, 314

Elbaz, D., Aussel, H., Cesarsky, C. J., et al. 1999, in ESA SP-427: The Universe as Seen by ISO, 427, 999

Epchtein, N., de Batz, B., Capoani, L., et al. 1997, The Messenger, 87, 27

Epchtein, N., Le Bertre, T., \& Lepine, J. R. D. 1990, A\&A, 227, 82

Epchtein, N., Le Bertre, T., Lepine, J. R. D., et al. 1987, A\&AS, 71, 39

Fluks, M. A., Plez, B., The, P. S., et al. 1994, A\&AS, 105, 311

Gail, H.-P., \& Sedlmayr, E. 1999, A\&A, 347, 594

Gilman, R. C. 1969, ApJ, 155, L185

Gilman, R. C. 1972, ApJ, 178, 423

Girardi, L. 1999, MNRAS, 308, 818

Girardi, L., \& Bertelli, G. 1998, MNRAS, 301, 149

Girardi, L., Bertelli, G., Bressan, A., et al. 2002, A\&A, 391, 195

Griffin, I.-P. 1993, MNRAS, 260, 831

Groenewegen, M., \& de Jong, T. 1993, A\&A, 267, 410

Groenewegen, M. A. T. 1995, A\&A, 293, 463

Groenewegen, M. A. T., Whitelock, P. A., Smith, C. H., \& Kerschbaum, F. 1998, MNRAS, 293, 18

Guglielmo, F., Epchtein, N., Arditti, F., \& Sevre, F. 1997, A\&AS, 122, 489

Guglielmo, F., Epchtein, N., Le Bertre, T., et al. 1993, A\&AS, 99, 31

Guglielmo, F., Le Bertre, T., \& Epchtein, N. 1998, A\&A, 334, 609

Habing, H. J. 1996, A\&ARv, 7, 97

Habing, H. J., Tignon, J., \& Tielens, A. G. G. M. 1994, A\&A, 286, 523 
Hackwell, J. A. 1972, A\&A, 21, 239

Hashimoto, O. 1995, ApJ, 442, 286

Hony, S., Waters, L. B. F. M., \& Tielens, A. G. G. M. 2002, A\&A, 390, 533

Ivezic, Z., \& Elitzur, M. 1994, in Cool Stars, Stellar Systems, and the Sun, 8, ASP Conf. Ser., 64, 711

Ivezic, Z., \& Elitzur, M. 1995, ApJ, 445, 415

Ivezic, Z., \& Elitzur, M. 1997, MNRAS, 287, 799

Jaeger, C., Molster, F. J., Dorschner, J., et al. 1998, A\&A, 339, 904

Kemper, F., Waters, L., de Korter, A., \& Tielens, A. 2001, A\&A, 369, 102

Lançon, A., \& Mouhcine, M. 2002, A\&A, 393, 167

Landau, L. D., \& Lifshitz, E. M. 1960, in Electrodynamics of Continuous Media (Pergamon Press), 460

Le Squeren, A. M., Sivagnanam, P., Dennefeld, M., \& David, P. 1992, A\&A, 254, 133

Leitherer, C., Alloin, D., von Alvensleben, U. F., et al. 1996, PASP, 108, 996

Lejeune, T., Cuisinier, F., \& Buser, R. 1998, A\&AS, 130, 65

Lepine, J. R. D., Ortiz, R., \& Epchtein, N. 1995, A\&A, 299, 453

Lewis, B. M. 1997, ApJS, 109, 489

Lewis, B. M., Eder, J., \& Terzian, Y. 1990, ApJ, 362, 634

Li, A., \& Mayo Greenberg, J. 2002, in Solid State Astrochemistry, ed. V. Pirronello, \& J. Krelowski (Kluwer), 1

Lorenz-Martins, S., de Araújo, F. X., Codina Landaberry, S. J., de Almeida, W. G., \& de Nader, R. V. 2001, A\&A, 367, 189

Lorenz-Martins, S., \& Lefevre, J. 1994, A\&A, 291, 831

Lorenz-Martins, S., \& Pompeia, L. 2000, MNRAS, 315, 856

Loup, C., Forveille, T., Omont, A., \& Paul, J. F. 1993, A\&AS, 99, 291

Marigo, P. 2002, A\&A, 387, 507

Marigo, P., Bressan, A., \& Chiosi, C. 1996, A\&A, 313, 545

Marigo, P., Girardi, L., \& Bressan, A. 1999, A\&A, 344, 123

Martin, C., Friedman, P., Schiminovich, D., et al. 1997, Am. Astron. Soc. Meet., 29, 1309

Mathis, J. S., Rumpl, W., \& Nordsieck, K. H. 1977, ApJ, 217, 425

Mouhcine, M. 2002, A\&A, 394, 125

Mouhcine, M., \& Lançon, A. 2002, A\&A, 393, 149

Nenkova, M., Ivezic, Z., \& Elitzur, M. 1999, in Workshop on Thermal Emission Spectroscopy and Analysis of Dust, Disks, and Regoliths, 3020

Neugebauer, G., Habing, H. J., van Duinen, R., et al. 1984, ApJ, 278, L1

Olivier, E. A., Whitelock, P., \& Marang, F. 2001, MNRAS, 326, 490

Ossenkopf, V., Henning, T., \& Mathis, J. S. 1992, A\&A, 261, 567

Pégourié, B. 1988, A\&A, 194, 335

Persson, S. E., Aaronson, M., Cohen, J. G., Frogel, J. A., \& Matthews, K. 1983, ApJ, 266, 105

Pietrzynski, G., \& Udalski, A. 2000, Acta Astron., 50, 337
Posch, T., Kerschbaum, F., Mutschke, H., et al. 1999, A\&A, 352, 609 Pretto, T. 2002, Master Thesis, University of Padova, Italy

Puget, J.-L., Abergel, A., Bernard, J.-P., et al. 1996, A\&A, 308, L5

Reimers, D. 1975, Mem. Soc. R. Sci. Liege, Ser., 6 (8), 369

Renzini, A., \& Buzzoni, A. 1983, Mem. Soc. Astron. It., 54, 739

Rowan-Robinson, M. 1980, ApJS, 44, 403

Rowan-Robinson, M., \& Harris, S. 1982, MNRAS, 200, 197

Salpeter, E. E. 1955, ApJ, 121, 161

Silva, L., Granato, G. L., Bressan, A., \& Danese, L. 1998, ApJ, 509, 103

Skrutskie, M. F., Schneider, S. E., Stiening, R., et al. 1997, in The Impact of Large Scale Near-IR Sky Surveys, ASSL, 210, 25

Sloan, G. C., Levan, P. D., \& Little-Marenin, I. R. 1996, ApJ, 463, 310

Speck, A. K., Barlow, M. J., \& Skinner, C. J. 1997, MNRAS, 288, 431

Speck, A. K., Hofmeister, A. M., \& Barlow, M. J. 1999, ApJ, 513, L87

Steffen, M., \& Schönberner, D. 2000, A\&A, 357, 180

Suh, K.-W. 1997, MNRAS, 289, 559

Suh, K.-W. 1999, MNRAS, 304, 389

Suh, K.-W. 2000, MNRAS, 315, 740

Suh, K.-W. 2002, MNRAS, 332, 513

Suh, K.-W., \& Jones, T. J. 1997, ApJ, 479, 918

Sylvester, R. J., Kemper, R., Barlow, M. J., et al. 1999, A\&A, 352, 587

Tantalo, R. 1998, Ph.D. Thesis, Univ. of Padova

Tantalo, R., Chiosi, C., Bressan, A., Marigo, P., \& Portinari, L. 1998, A\&A, 335, 823

Tinsley, B. M. 1980, Fund. Cos. Phys., 5, 287

van der Veen, W. E. C. J., \& Habing, H. J. 1988, A\&A, 194, 125

van der Veen, W. E. C. J., Omont, A., Habing, H. J., \& Matthews, H. E. 1995, A\&A, 295, 445

van Loon, J. T. 2000, A\&A, 354, 125

van Loon, J. T., Groenewegen, M. A. T., de Koter, A., et al. 1999, A\&A, 351, 559

van Loon, J. T., Zijlstra, A. A., Whitelock, P. A., et al. 1998, A\&A, 329,169

Vassiliadis, D. A., \& Wood, P. R. 1993, ApJ, 413, 641

Vassiliadis, E., \& Wood, P. R. 1993, ApJ, 413, 641

Volk, K., \& Kwok, S. 1988, ApJ, 331, 435

Volk, K., Kwok, S., \& Langill, P. P. 1992, ApJ, 391, 285

Waters, L. B. F. M., \& Molster, F. G. 1999, in Asymptotic Giant Branch Stars, IAU Symp., 191, 209

Waters, L. B. F. M., Molster, F. J., de Jong, T., et al. 1996, A\&A, 315, L361

Weingartner, J. C., \& Draine, B. T. 2001, ApJ, 548, 296

Whitelock, P., \& Feast, M. 1994, Ap\&SS, 217, 153

Xiong, G. Z., Chen, P. S., \& Gao, H. 1994, A\&AS, 108, 661

York, D. G., Adelman, J., Anderson, J. E., et al. 2000, AJ, 120, 1579 